\title{
ON THE CAUCHY PROBLEM AND INITIAL TRACES FOR A DEGENERATE PARABOLIC EQUATION
}

\author{
E. DI BENEDETTO AND M. A. HERRERO
}

Abstract. We consider the Cauchy problem

$$
\begin{cases}u_{t}-\operatorname{div}\left(|D u|^{p-2} D u\right)=0 & \text { in } \mathbf{R}^{N} \times(0, \infty), p>2, \\ u(x, 0)=u_{0}(x), & x \in \mathbf{R}^{N},\end{cases}
$$

and discuss existence of solutions in some strip $S_{T} \equiv \mathbf{R}^{N} \times(0, T), 0<T \leq \infty$, in terms of the behavior of $x \rightarrow u_{0}(x)$ as $|x| \rightarrow \infty$. The results obtained are optimal in the class of nonnegative locally bounded solutions, for which a Harnack-type inequality holds. Uniqueness is shown under the assumption that the initial values are taken in the sense of $L_{\text {loc }}^{1}\left(\mathbf{R}^{N}\right)$.

\section{INTRODUCTION}

A classical result of Tychonov [14] states that the Cauchy problem for the heat equation is uniquely solvable for continuous initial data $x \rightarrow u_{0}(x)$ satisfying the growth condition

$$
\left|u_{0}(x)\right| \leq C \exp \left(a|x|^{2}\right), \quad \text { as }|x| \rightarrow \infty,
$$

for some positive constants $C, a$. In such a case, the solution $u$ exists in the strip $\mathbf{R}^{N} \times\left(0, \frac{a}{4}\right)$. The growth condition is optimal in the sense that every nonnegative solution of the heat equation in $\mathbf{R}^{N} \times(0, T), 0<T<\infty$, has a unique $\sigma$-finite Borel measure $\mu$ as initial trace, satisfying (see $[15,2]$ )

$$
\int_{\mathbf{R}^{N}} \exp \left\{-|x|^{2} / 4 T\right\} d \mu<\infty .
$$

In this note we consider the Cauchy problem for the nonlinear version of the heat equation

$$
u_{t}-\operatorname{div}\left(|D u|^{p-2} D u\right)=0 \quad \text { in } S_{T} \equiv \mathbf{R}^{N} \times(0, T), \quad p>2,
$$

and address the issue of growth conditions on the initial datum $u_{0}(\cdot)$ as $|x| \rightarrow$ $\infty$ for a unique solution to exist, as well as the optimality of such a growth. A rough description of our results is the following.

Received by the editors October 19, 1987.

1980 Mathematics Subject Classification (1985 Revision). Primary 35K55.

Key words and phrases. Nonlinear degenerate parabolic equations, optimal conditions for existence, Harnack inequality.

The authors were partially supported, respectively, by NSF grant DMS-8502297 and CICYT grant PB86-0112-C02-02. 
If $u_{0} \in L_{\text {loc }}^{\infty}\left(\mathbf{R}^{N}\right)$ and

$$
\left|u_{0}(x)\right| \leq c_{0}|x|^{p /(p-2)} \quad \text { as }|x| \rightarrow \infty
$$

for some $c_{0}>0$, then the Cauchy problem associated with (1.1) is uniquely solvable (in a weak sense specified below) in the strip $S_{T}, T=C(N, p) / c_{0}^{p-2}$, where $C(N, p)$ is a universal constant depending only on $N$ and $p$.

In fact $u_{0}$ does not have to be locally bounded (see $\S 2$ ). It could be in $L_{\text {loc }}^{1}\left(\mathbf{R}^{N}\right)$ or even a $\sigma$-finite Borel measure in $\mathbf{R}^{N}$, with (1.2) suitably rephrased in terms of integral averages.

The growth condition (1.2) is optimal in the sense that a nonnegative solution of (1.1) in $S_{T}$ determines uniquely an initial datum (the initial trace) which is a $\sigma$-finite Borel measure $\mu$ in $\mathbf{R}^{N}$ satisfying

$$
\begin{gathered}
f_{|x|<p} d \mu \leq C\left\{\left(\frac{\rho^{p}}{T}\right)^{1 /(p-2)}+\left(\frac{T}{\rho^{p}}\right)^{N / p}[u(0, T)]^{\kappa / p}\right\}, \\
\kappa=N(p-2)+p,
\end{gathered}
$$

for some positive constant $C=C(N, p)$. Here and in what follows

$$
f_{|x|<\rho} d \mu=\rho^{-N} \int_{|x|<\rho} d \mu .
$$

In one space dimension $N=1$, the problem has been studied by Kalashnikov $[11,12]$, who proved existence of a unique solution of $(1.1)$ in $S_{T}$, for some (small) $T$, if the initial datum satisfies

$$
\left|u_{0}(x)\right| \leq C\left(1+|x|^{2}\right)^{p / 2(p-2)}, \quad \forall x \in \mathbf{R}
$$

for some $C>0$.

For the porous medium equation

$$
u_{t}-\Delta|u|^{m-1} u=0, \quad m>1,
$$

the problem of growth condition on the initial datum $u_{0}$ has been considered by Bènilan-Crandall-Pierre [5], the optimality of such a growth by AronsonCaffarelli [3], and uniqueness of solutions for data $\mu$ satisfying the analog of (1.3) by Dahlberg-Kenig [6]. Our approach is different from that in the quoted papers and in fact our methods yield generalizations also in the case of (1.6).

We will discuss later how our results relate to the cited ones and what are the possible extensions.

\section{THE RESULTS}

Consider the Cauchy problem

$$
\begin{gathered}
\frac{\partial}{\partial t} u-\operatorname{div}\left(|D u|^{p-2} D u\right)=0 \quad \text { in } S_{T}, \quad p>2 \\
u(\cdot, 0)=u_{0}(\cdot) \in L_{\text {loc }}^{1}\left(\mathbf{R}^{N}\right)
\end{gathered}
$$


A measurable function $(x, t) \rightarrow u(x, t)$ defined in $S_{T}$ is a weak solution of (2.1), (2.2) if for every bounded open set $\Omega \subset \mathbf{R}^{N}$,

$$
\begin{aligned}
& u \in C\left(0, T ; L^{1}(\Omega)\right) \cap L^{p-1}\left(0, T ; W^{1, p-1}(\Omega)\right), \quad \text { and } \\
& \int_{\Omega} u(x, t) \varphi(x, t) d x+\int_{0}^{t} \int_{\Omega}\left\{-u \varphi+|D u|^{p-2} D u D \varphi\right\} d x d \tau \\
&=\int_{\Omega} u_{0}(x) \varphi(x, 0) d x
\end{aligned}
$$

for all $0<t<T$ and all testing functions

$$
\varphi \in W^{1, \infty}\left(0, T ; L^{\infty}(\Omega)\right) \cap L^{\infty}\left(0, T ; W_{0}^{1, \infty}(\Omega)\right) .
$$

Weak subsolutions (resp. supersolutions) are defined as above except that in (2.3) equality is replaced by $\leq$ (resp. $\geq$ ) and $\varphi$ is taken to be nonnegative.

If $(2.2)$ is replaced by

$$
u(\cdot, 0)=\mu
$$

where $\mu$ is a $\sigma$-finite Borel measure $\mathbf{R}^{N}$, then we say that $u$ is a weak solution of (2.1), (2.5) if for every bounded open set $\Omega \subset \mathbf{R}^{N}$ and $\forall t \in(0, T), u$ satisfies (2.3) with the right-hand side replaced by

$$
\int_{\Omega} \varphi(x, 0) d \mu
$$

$\forall \varphi \in C^{1}\left(\bar{\Omega}_{T}\right)$ such that $x \rightarrow \varphi(x, t)$ is compactly supported in $\Omega \forall t \in[0, T]$.

As a way of measuring the growth of a function $f \in L_{\mathrm{loc}}^{1}\left(\mathbf{R}^{N}\right)$ as $|x| \rightarrow \infty$ we set

$$
\left\||| f\left|\|_{r}=\sup _{\rho \geq r} \rho^{-\kappa /(p-2)} \int_{B_{\rho}}\right| f \mid d x, \quad r>0, \kappa=N(p-2)+p, p>2,\right.
$$

where $B_{\rho} \equiv\left\{x \in \mathbf{R}^{N}|| x \mid<\rho\right\}$. If $\mu$ is a $\sigma$-finite Borel measure in $\mathbf{R}^{N}$, set

$$
\left\||| \mu\left|\|_{r}=\sup _{\rho \geq r} \rho^{-\kappa /(p-2)} \int_{B_{\rho}}\right| d \mu \mid,\right.
$$

where $|d \mu|$ is the variation of $\mu$.

Remarks. (i) $\||f|\|_{r}<+\infty, \forall r>0$ if $f \in L^{1}\left(\mathbf{R}^{N}\right)$.

(ii) \|\|$\mu \|_{r}<+\infty, \forall r>0$ if and only if $\|\mu\| \|_{r_{0}}<\infty$ or some $r_{0}>0$.

With $C_{i}=C_{i}\left(A_{1}, A_{2}, \ldots\right)$ we denote positive constants that can be quantitatively determined a priori only in dependence on the specified arguments.

2-(i). Existence.

Theorem 1. For every $\sigma$-finite Borel measure $\mu$ in $\mathbf{R}^{N}$ satisfying

$$
\|\| \mu \mid \|_{r}<\infty, \text { for some } r>0 \text {, }
$$


there exists a weak solution $u$ of (2.1), (2.5) in $S_{T(\mu)}$, where

$$
T(\mu)= \begin{cases}C_{0}\left[\lim _{r \rightarrow \infty}\left|\|\mu \mid\|_{r}\right]^{-(p-2)}\right. & \text { if } \lim _{r \rightarrow \infty}\||| \mu \mid\|_{r}>0, \\ +\infty & \text { if } \lim _{r \rightarrow \infty}\|\mu \mid\|_{r}=0\end{cases}
$$

and $C_{0}=C_{0}(N, p)$.

Let

$$
T_{r}(\mu)=C_{0}\left[|||\mu| \mid \|_{r}\right]^{-(p-2)} .
$$

Then $\forall 0<t<T_{r}(\mu), \forall \rho \geq r>0$,

$$
\begin{aligned}
\|u(\cdot, t) \mid\|_{r} & \leq C_{1}\|\mu\| \|_{r}, \\
\|u(\cdot, t)\|_{\infty, B_{\rho}} & \leq C_{2} t^{-N / \kappa} \rho^{p /(p-2)}\|\mu\| \|_{r}^{p / \kappa}, \\
\|D u(\cdot, t)\|_{\infty, B_{\rho}} & \leq C_{3} t^{-(N+1) / \kappa} \rho^{2 /(p-2)}\|\| \mu \|_{r}^{2 / \kappa},
\end{aligned}
$$

where $C_{i}=C_{i}(N, p), i=1,2,3$.

For every bounded open set $\Omega \subset \mathbf{R}^{N}$ and $\forall \varepsilon>0$

$$
\exists C_{4}=C_{4}(N, p, \varepsilon, \operatorname{diam} \Omega), \quad C_{5}=C_{5}(N, p, \varepsilon)
$$

such that

$$
\begin{aligned}
\int_{0}^{t} \int_{\Omega}|D u|^{q} d x d \tau \leq & C_{4}\|\| \mu \|_{r}^{C_{5}}, \\
& \text { where } q=p-(N+\varepsilon) /(N+1), \forall 0<t<T_{r}(\mu) .
\end{aligned}
$$

In particular if $\varepsilon=1$

$$
\int_{0}^{t} \int_{\Omega}|D u|^{p-1} d x d \tau \leq C_{6} t^{1 / \kappa}\left|\|\mu \mid\|_{r}^{1+(p-2) / \kappa},\right.
$$

where $C_{6}=C_{6}(N, p, \Omega)$. Further if $\Omega \equiv B_{\rho}, \rho \geq r$,

$$
\int_{0}^{t} \int_{B_{\rho}}|D u|^{p-1} d x d \tau \leq C_{7} t^{1 / \kappa} \rho^{1+\kappa /(p-2)} \mid\|\mu\|_{r}^{1+(p-2) / \kappa},
$$

where $C_{7}=C_{7}(N, p)$.

Finally, $(x, t) \rightarrow D u(x, t)$ is Hölder continuous in $\bar{\Omega} \times[\eta, T(\mu)-\eta], 0<$ $\eta<T(\mu)$, with Hölder constants and exponents depending upon $N, p, C_{i}$, $i=0,1, \ldots, 4, \operatorname{diam} \Omega, \eta,\|\mu\| \|_{r}$.

The functional dependence of our estimates is optimal as shown by the following two explicit solutions of (2.1).

$$
\mathscr{D}(x, t)=\left\{A\left(\frac{T}{T-t}\right)^{\frac{N(p-2)}{\kappa(p-1)}}+\left(\frac{p-2}{p-1}\right) \kappa^{\frac{1}{p-1}}\left(\frac{|x|^{p}}{T-t}\right)^{\frac{1}{p-1}}\right\}^{\frac{p-1}{p-2}},
$$

where $A, T$ are two positive parameters. 
A quick calculation shows that

$$
\lim _{r \rightarrow \infty}\|\| \mathscr{D}(\cdot, 0)\|\|_{r}=\frac{1}{N}\left(\frac{p-2}{p-1}\right)^{(p-1) /(p-2)}\left(\frac{\kappa}{T}\right)^{1 /(p-2)} .
$$

Therefore $\mathscr{D}(x, t)$ exists up to the blowup time

$$
T=\frac{\kappa}{N^{p-2}}\left(\frac{p-2}{p-1}\right)^{p-1}\left\{\lim _{r \rightarrow \infty}\|\| \mathscr{D}(\cdot, 0) \|_{r}\right\}^{-(p-2)}
$$

The second explicit solution is (see [16])

$$
\begin{array}{rlrl}
\mathscr{B}(x, t) & =t^{-N / \kappa}\left\{1-\gamma_{p}\left(\frac{|x|}{t^{1 / \kappa}}\right)^{p /(p-1)}\right\}_{+}^{(p-1) /(p-2)}, & t>0, \\
\gamma_{p}=\left(\frac{1}{\kappa}\right)^{1 /(p-1)} \frac{p-2}{p}, & p>2 .
\end{array}
$$

The functions $\mathscr{B}$ solves $(2.1)$ with initial datum $\mu=M \delta_{0}$, where $\delta_{0}$ is the Dirac measure at zero and $M \equiv\|\mathscr{B}(\cdot, t)\|_{1, \mathbf{R}^{N}}, t>0$.

For $t>0,\|\mathscr{B}(\cdot, t)\|_{\infty, \mathbf{R}^{N}} \leq t^{-N / \kappa}$, and

$$
\|D \mathscr{B}(\cdot, t)\|_{\infty, \mathbf{R}^{N}} \leq t^{-(N+1) / \kappa} \frac{p}{p-1} \gamma_{p}^{(p-1) / p} .
$$

Moreover $|D \mathscr{B}| \in L^{q}\left(\mathbf{R}^{N} \times[0, s]\right), s>0, q=p-(N+\varepsilon) /(N+1), \forall \varepsilon>0$, but $|D \mathscr{B}| \notin L^{q_{0}}\left(\mathbf{R}^{N} \times[0, s]\right), q_{0}=p-N /(N+1)$.

For initial data $u_{0} \in L^{1}\left(\mathbf{R}^{N}\right)$ estimates (2.10), (2.11) agree with those of [10] and [1], respectively.

2-(ii). Harnack inequality and initial traces. Consider solutions of (2.1) with no further reference to initial data. These are measurable functions $u$ defined in $S_{T}, 0<T \leq \infty$, such that for every bounded open set $\Omega \subset \mathbf{R}^{N}$ and $\forall \varepsilon \in(0, T)$

$$
\begin{gathered}
u \in C\left(\varepsilon, T ; L^{p}(\Omega)\right) \cap L^{p}\left(\varepsilon, T ; W^{1, p}(\Omega)\right), \\
\int_{\Omega} u(x, t) \varphi(x, t) d x+\int_{0}^{t} \int_{\Omega}\left\{-u \varphi_{t}+|D u|^{p-2} D u D \varphi\right\} d x d \tau=0, \\
\forall t \in(\varepsilon, T), \forall \varphi \in W^{1, \infty}\left(0, T ; L^{\infty}(\Omega)\right) \cap L^{\infty}\left(\varepsilon, T ; W_{0}^{1, \infty}(\Omega)\right), \\
t \rightarrow \varphi(\cdot, t)=0,0<t \leq \varepsilon .
\end{gathered}
$$

Remark 2.1. From (2.15) and [7, 9] it follows that $(x, t) \rightarrow D u(x, t)$ is Hölder continuous in every compact subset of $\mathbf{R}^{N} \times(0, T)$. These solutions belong to the same regularity class of those found in Theorem 1 .

Theorem 2. Let $u$ be a nonnegative solution of (2.1) in $S_{T}$ for some $0<T \leq$ $+\infty$. There exists a unique $\sigma$-finite Borel measure $\mu$ on $\mathbf{R}^{N}$ such that

$$
\lim _{t \backslash 0} \int_{\mathbf{R}^{N}} u(x, t) \varphi(x) d x=\int_{\mathbf{R}^{N}} \varphi d \mu,
$$

for all $\varphi$ continuous and compactly supported in $\mathbf{R}^{N}$. 
Moreover $\forall R>0, \forall 0<t \leq T$

$$
R^{-N} \int_{B_{R}} d \mu \leq \gamma\left\{\left(\frac{R^{p}}{t}\right)^{1 /(p-2)}+\left(\frac{t}{R^{p}}\right)^{N / p}[u(0, t)]^{\kappa / p}\right\},
$$

where $\kappa=N(p-2)+p$, and $\gamma=\gamma(N, p)$.

The existence part of Theorem 2 is an immediate consequence of the Harnack inequality, which we state as a theorem in view of its independent interest.

Theorem 3. Let $u$ be a nonnegative solution of (2.1) in $S_{T}$. There exists a constant $\gamma=\gamma(N, p)$ such that $\forall R>0, \forall 0<\tau \leq T / 2$

$$
f_{B_{R}} u(x, \tau) d x \leq \gamma\left\{\left(\frac{R^{p}}{T}\right)^{1 /(p-2)}+\left(\frac{T}{R^{p}}\right)^{N / p}[u(0, T)]^{\kappa / p}\right\} .
$$

The uniqueness of $\mu$ will make essential use of estimate (2.14).

\section{2-(iii). Uniqueness.}

Theorem 4. Let $u, v$ be two solutions of (2.1) in $S_{T}$, for some $0<T \leq+\infty$, in the sense of (2.15), (2.16), such that

$$
\sup _{(0, T)}\|\| u(\cdot, t)\|\|_{r}, \quad \sup _{(0, T)}\|v(\cdot, t) \mid\|_{r}<\infty
$$

for some $r>0$, and

$$
\lim _{t \searrow 0}(u(\cdot, t)-v(\cdot, t))=0 \quad \text { in } L_{\mathrm{loc}}^{1}\left(\mathbf{R}^{N}\right) .
$$

Then $u \equiv v$ in $S_{T}$.

Remarks. (i) In Theorem 4, $u, v$ are not required to be nonnegative.

(ii) The solutions constructed in Theorem 1 with initial data $\mu \in L_{\text {loc }}^{1}\left(\mathbf{R}^{N}\right)$ satisfy the assumption. Therefore Theorem 4 could be rephrased as a uniqueness theorem for initial data $u_{0} \in L_{\text {loc }}^{1}\left(\mathbf{R}^{N}\right)$ and $\left\|u_{0}\right\|_{r}<\infty, r>0$.

(iii) Uniqueness of nonnegative solutions for initial datum a positive $\sigma$-finite Borel measure $\mu$ would follow by the approximation argument of [6] if one knew that uniqueness holds for initial datum $\mu$ satisfying $\int_{\mathbf{R}^{\prime}} d \mu<\infty$.

We have been unable to prove this last statement.

2-(iv). Nonnegative solutions. Let $u \geq 0$ be a weak solution of (2.1) in $S_{T}$ in the sense of (2.15), (2.16). The Harnack inequality of Theorem 3 implies that $\forall r>0$

$$
\sup _{0<t \leq T}\|\| u(\cdot, t) \| \mid \leq \gamma T^{-1 /(p-2)}\left\{1+\left(T^{1 /(p-2)} \frac{u(0, T)}{r^{p}}\right)^{\kappa / p}\right\} \equiv \Lambda .
$$

Such an a priori bound permits us to prove the following theorem.

Theorem 5. Every nonnegative solution of (2.1) in $S_{T}$ grows at most as $|x|^{p /(p-2)}$ when $|x| \rightarrow \infty$. Precisely

$$
\|u(\cdot, t)\|_{\infty, B_{\rho}} \leq \gamma t^{-N / \kappa} \rho^{p /(p-2)} \Lambda^{p / \kappa}, \quad \forall 0<t \leq T, \forall \rho \geq r>0, \forall r>0 .
$$

The theorem is proved in $\S 7$. 
2-(v). Methods and extensions. Given a $\sigma$-finite Borel measure $\mu$ in $\mathbf{R}^{N}$ satisfying $\|\mu \mu\|_{r}<\infty$ for some $r>0, \exists x \rightarrow u_{0, n}(x) \in C_{0}^{\infty}\left(\mathbf{R}^{N}\right)$ such that $\forall \varphi \in C_{0}\left(\mathbf{R}^{N}\right)$

$$
\int_{\mathbf{R}^{N}} u_{0, n} \varphi d x \rightarrow \int_{\mathbf{R}^{N}} \varphi d \mu
$$

and $\left\|\left|u_{0, n}\right|\right\|_{r} \rightarrow\|\| \mu\|\|_{r}, \forall r>0$.

The Cauchy problem (2.1) with initial datum $u_{0, n}$ has a unique solution $u_{n}$ global in time (see $\left.[4,10]\right)$. If we can prove estimates (2.9)-(2.14) for $u_{n}$ with \|\|$\mu \mid \|_{r}$ replaced by \|\|$u_{0, n} \mid \|_{r}$, Theorem 1 will follow by a standard limiting process, modulo the compactness results of [7,9]. Henceforth we will refer to the proof of Theorem 1 as to the proof of (2.9)-(2.14) for the unique solution $u$ of (2.1) with initial datum $u_{0} \in C_{0}^{\infty}\left(\mathbf{R}^{N}\right)$. Our proofs in $\S \S 3,4$ show that it is not restrictive to assume $u_{0} \geq 0$ (and hence $u \geq 0$ ); in fact (2.9), (2.10), (2.12)-(2.14) hold for nonnegative subsolutions of (2.1).

Estimate (2.10) is in the spirit of a similar one of [5] for solutions of the porous medium equation (1.6). In that work the key estimates were of elliptic nature in view of the "semiconvexity inequality"

$$
u_{t} \geq-k u / t ; \quad k \text { a constant }
$$

valid for weak solutions $u \geq 0$ of (1.6) in $\mathbf{R}^{N} \times(0, T)$ (see [4]) and strongly linked to the homogeneity of the principal part of theoperator in (1.6).

There is a basic difference between the nonlinearity exhibited by (2.1) and the nonlinearity of (1.6). Such a difference becomes apparent in estimate (2.10). Working with (1.6) one can relate quite easily the quantity $\int_{B_{\rho}} u(x, t) d x, t>0$ with the analogous quantities $\int_{B_{2 \rho}} u(x, \tau) d x$ for $\tau \in(0, t)$; this is because the Laplacian permits a double integration by parts. It is this fact that makes estimates like $(2.10)$ relatively straightforward once a bound for $\left\|u^{m-1}\right\|_{\infty, B_{p}}$ has been derived.

In the case of (2.1) one is forced to find in addition to (2.10) a rather delicate estimate of

$$
\int_{0}^{t} \int_{B_{\rho}}|D u|^{p-1} d x d \tau,
$$

in terms of $\int_{0}^{t}\|\| u(\cdot, \tau) \mid \|_{r} d \tau$. This is what makes possible the proof of (2.14), which in turn is the key estimate for Theorems 2 and 4.

Even though regularizing effects like (2.17) hold for (2.1) (see [4]) we have avoided them. Our estimates are of parabolic nature and flexible enough to generalize $(2.9),(2.10),(2.12)-(2.14)$ to nonnegative weak subsolutions of

$$
\begin{gathered}
\frac{\partial}{\partial t} u-\operatorname{div} \mathbf{A}(x, t, u, D u) \leq B(x, t, u, D u), \\
u(x, 0)=u_{0}(x) \in L_{\text {loc }}^{1}\left(\mathbf{R}^{N}\right), \quad\left\|u_{0}\right\|_{r}<\infty \text { for some } r>0,
\end{gathered}
$$

provided they can be approximated by bounded solutions. 
The structure conditions in (2.18) are

$$
\begin{gathered}
\lambda_{0}|D u|^{p}-g_{0}(x, t) \leq \mathbf{A}(x, t, u, D u) \cdot D u \leq \Lambda_{0}|D u|^{p}+g_{1}(x, t), \\
|B(x, t, u, D u)| \leq B_{0}|D u|^{p-1}+g_{2}(x, t),
\end{gathered}
$$

where $0<\lambda_{0}<\Lambda_{0}, B_{0}>0$ are given constants and $g_{i}, i=0,1,2$, are given bounded functions in $\mathbf{R}^{N+1}$.

Moreover, (2.11) also holds true if some differentiability is imposed on the vector field $\mathbf{A}: \mathbf{R}^{2 N+2} \rightarrow \mathbf{R}^{N}$ and the function $B: \mathbf{R}^{2 N+2} \rightarrow \mathbf{R}$, and if (2.18) holds with equality replacing the inequality. We refer to $[7,9]$ for the precise differentiability assumptions on $\mathbf{A}$ and $B$.

The ingredients in the proof of Theorem 3 are the gradient estimate (2.14) and the following "intrinsic" Harnack estimate.

Theorem 1 of [8]. Let $u \geq 0$ be a local weak solution of (1.1) in some cylindrical domain $\Omega_{T} \equiv \Omega \times(0, T)$. Let $\left(x_{0}, t_{0}\right) \in \Omega_{T}$, let $B_{\rho}\left(x_{0}\right)$ be the ball of radius $\rho$ about $x_{0}$, and assume $u\left(x_{0}, t_{0}\right)>0$. There exist two constants $\bar{C}_{i}=\bar{C}_{i}(N, p)$, $i=0,1$ such that

$$
u\left(x_{0}, t_{0}\right) \leq \bar{C}_{0} \inf _{x \in B_{\rho}\left(x_{0}\right)} u\left(x, t_{0}+\bar{C}_{1} \frac{\rho^{p}}{u^{p-2}\left(x_{0}, t_{0}\right)}\right),
$$

provided the box

$$
Q_{0} \equiv B_{2 \rho}\left(x_{0}\right) \times\left\{t_{0}-\bar{C}_{1} \frac{\rho^{p}}{u^{p-2}\left(x_{0}, t_{0}\right)}, t_{0}+\bar{C}_{1} \frac{\rho^{p}}{u^{p-2}\left(x_{0}, t_{0}\right)}\right\}
$$

is all contained in $\Omega_{T}$.

It was observed in [8] that (2.19) implies $\forall \theta>0$

$$
u\left(x_{0}, t_{0}\right) \leq \gamma\left\{\left(\frac{\rho^{p}}{\theta}\right)^{1 /(p-2)}+\left(\frac{\theta}{\rho^{p}}\right)^{N / p}\left[\int_{B_{\rho}\left(x_{0}\right)} u\left(x, t_{0}+\theta\right)\right]^{\kappa / p}\right\}
$$

for a constant $\gamma=\gamma(N, p)$, provided the box

$$
Q(\rho, \theta) \equiv B_{\rho}\left(x_{0}\right) \times\left\{t_{0}-\bar{C}_{1} \theta, t_{0}+\bar{C}_{1} \theta\right\}
$$

is all contained in the domain of definition of $u$.

Recall that local solutions of (1.1) are Hölder continuous [7] and therefore $u(x, t)$ is well defined $\forall(x, t) \in \Omega_{T}$.

In view of the continuity of $u$, within the ball $B_{\rho}\left(x_{0}\right)$ there exists at least one $\bar{x}$ such that

$$
f_{B_{\rho}\left(x_{0}\right)} u\left(x, t_{0}\right) d x=u\left(\bar{x}, t_{0}\right) .
$$

Therefore (2.20) implies

$$
f_{B_{\rho}\left(x_{0}\right)} u\left(x, t_{0}\right) d x \leq \gamma\left\{\left(\frac{\rho^{p}}{\theta}\right)^{1 /(p-2)}+\left(\frac{\theta}{\rho^{p}}\right)^{N / p}\left[u\left(x_{0}, t_{0}+\theta\right)\right]^{\kappa / p}\right\}
$$


$\forall \rho>0, \forall \theta>0$ provided the box $Q(\rho, \theta)$ is all contained in the domain of definition of $u$. This last requirement, when applied to nonnegative solutions of (2.1) in $S_{T} \equiv \mathbf{R}^{N} \times(0, T)$, says that $t_{0}$ and $\theta$ must satisfy

$$
0<t_{0}-\bar{C}_{1} \theta<t_{0}+\bar{C}_{1} \theta<T \text {. }
$$

Thus the main difference between Theorem 3 and (2.21) is that in Theorem 3 $t_{0}$ can be arbitrarily close to zero, whereas in (2.21) $t_{0}$ must be of the order of $T$.

It will be precisely (2.14) that will permit us to overcome this difficulty and extend (2.21) for all $t_{0} \in(0, T)$.

The existence part of Theorem 2 will follow from the Harnack estimate. Uniqueness of the initial trace $\mu$ is based on the following.

Lemma 2.2. Let $u \geq 0$ be any weak solution of (2.1) in $S_{T}$. Then $\forall R>0$, $\forall \varepsilon \in(0,1]$

$$
\begin{aligned}
& f_{B_{(1+\varepsilon) R}} u(x, t) d x \\
& \quad \geq f_{B_{R}} u(x, \tau) d x\left\{(1+\varepsilon)^{-N}-\frac{\gamma}{\varepsilon}\left(\left[f_{B_{R}} u(x, \tau) d x\right]^{p-2} R^{-p}(t-\tau)\right)^{1 / \kappa}\right\}
\end{aligned}
$$

for all $0<\tau<t \leq \gamma_{*} R^{p} /\left[f_{B_{R}} u(x, \tau) d x\right]^{p-2}$, where $\gamma, \gamma_{*}=\gamma, \gamma_{*}(N, p)$.

Lemmas of this kind for the porous medium equation, though less general, were referred to as lemmas on "how fast the material can escape a given ball" (see [3]). The proof of the analogous fact for solutions of (1.6) in [3] is rather complicated, and it is based on Alexandrov reflection technique. In our case it is a simple consequence of estimate (2.14).

Finally, the strength of (2.14) appears in the proof of Theorem 4. It implies that if $u$ and $v$ are solutions of $(2.1)$ in $S_{T}$ such that $u(\cdot, t)-v(\cdot, t) \equiv w(\cdot, t) \rightarrow$ 0 in $L_{\text {loc }}^{1}\left(\mathbf{R}^{N}\right)$ as $t \searrow 0$, then $\forall \varepsilon \in(0,1 / N)$ as $t \searrow 0, w(\cdot, t) \rightarrow 0$ in $L_{\text {loc }}^{1+\varepsilon}\left(\mathbf{R}^{N}\right)$. Once this is shown, Theorem 4 follows in a rather straightforward way.

\section{Proof of Theorem 1. The $L^{\infty}$-estimate}

Let $u \in L^{\infty}\left(0, T ; L^{1}\left(\mathbf{R}^{N}\right)\right) \cap L^{p}\left(0, T ; W^{1, p}(\Omega)\right), p>2$, be the unique solution of

$$
\begin{aligned}
& \frac{\partial}{\partial t} u-\operatorname{div}\left(|D u|^{p-2} D u\right)=0, \quad \mathbf{R}^{N}, t>0, \\
& u(x, 0)=u_{0}(x) \in C_{0}^{\infty}\left(\mathbf{R}^{N}\right) .
\end{aligned}
$$

By the results of [9], $D u \in C^{\alpha, \alpha / 2}(\Omega \times(\varepsilon, T))$ for any bounded open set $\Omega \subset \mathbf{R}^{N}$ and any $\varepsilon>0, T>0$ and some $\alpha \in(0,1), \alpha=\alpha\left(\varepsilon, T,\left\|u_{0}\right\|_{\infty},|\Omega|\right)$. Since $u_{t} \in L^{2}\left(0, T ; L_{\mathrm{loc}}^{2}\left(\mathbf{R}^{N}\right)\right), \forall 0<T<\infty,(3.1)$ holds a.e. $\mathbf{R}^{N} \times(0, \infty)$. 
Such regularity will suffice to justify the calculations to follow. We shall denote with $\gamma$ a generic positive constant depending only on $N, p$. The proof of (2.10) requires several steps.

3-(i). Preliminaries. Let $T>0, \rho>0$ be fixed and consider the sequences

$$
\begin{aligned}
T_{n}=\frac{T}{2}-\frac{T}{2^{n+1}}, \quad \rho_{n}=\rho+\frac{\rho}{2^{n+1}}, \quad \bar{\rho}_{n}=\frac{1}{2}\left(\rho_{n}+\rho_{n+1}\right) & =\rho+\frac{3 \rho}{2^{n+2}}, \\
n & =0,1,2, \ldots .
\end{aligned}
$$

Set

$$
B_{n}=B_{\rho_{n}}, \quad \bar{B}_{n}=B_{\rho_{n}}, \quad Q_{n} \equiv B_{n} \times\left(T_{n}, T\right), \quad \bar{Q}_{n} \equiv \bar{B}_{n} \times\left(T_{n+1}, T\right),
$$

and let $(x, t) \rightarrow \zeta_{n}(x, t)$ be a piecewise smooth cutoff function in $Q_{n}$ satisfying

$$
\begin{gathered}
\zeta_{n}(x, t)=1, \quad(x, t) \in \bar{Q}_{n} \text { and } \\
\left|D \zeta_{n}\right| \leq 2^{n+2} / \rho, \quad 0 \leq \partial \zeta_{n} / \partial t \leq 2^{n+2} / T .
\end{gathered}
$$

Finally, for a positive number $k$ we will consider the increasing sequence

$$
k_{n}=k-k / 2^{n+1}, \quad n=0,1,2, \ldots .
$$

Let $w \in L^{\infty}\left(0, T ; L^{s}\left(B_{\rho}\right)\right) \cap L^{m}\left(0, T ; W_{0}^{1, m}\left(B_{\rho}\right)\right), s, m>1$. By GagliardoNirenberg's inequality (see $[13$, p. 62]), for a.e. $t \in(0, T)$

$$
\|w\|_{q, B_{\rho}} \leq \gamma\|D w\|_{m, B_{\rho}}^{\eta}\|w\|_{s, B_{\rho}}^{1-\eta}
$$

where

$$
\eta=(1 / s-1 / q)(1 / N-1 / m+1 / s)^{-1}
$$

and $\gamma=\gamma(N, p)$. Choose $\eta=m / q$, take the $q$-power, and integrate over $(0, T)$. Then if $Q \equiv B_{\rho} \times(0, T)$

$$
\iint_{Q}|w|^{q} d x d \tau \leq \gamma\left(\iint_{Q}|D w|^{m} d x d t\right)\left(\underset{0 \leq t \leq T}{\operatorname{ess} \sup } \int_{B_{\rho} \times\{t\}}|w|^{s} d x\right)^{m / N}
$$

for a new constant $\gamma=\gamma(N, m, s)$.

In particular, for the choices

$$
m=p ; s=p^{2} / 2(p-1),
$$

we have $q=p(1+s / N)$, and

$$
\iint_{Q}|w|^{p(1+s / N)} d x d t \leq \gamma\left(\iint_{Q}|D w|^{p} d x d t\right)\left(\underset{0 \leq t \leq T}{\operatorname{ess} \sup } \int_{B_{\rho}}|w(t)|^{s} d x\right)^{p / N} .
$$

Also for the choices

$$
m=s=2 \text {, }
$$


we have $q=2(N+2) / N$, and

$$
\iint_{Q}|w|^{2+4 / N} d x d t \leq \gamma\left(\iint_{Q}|D w|^{2} d x d t\right)\left(\operatorname{esssup}_{0 \leq t \leq T} \int_{B_{\rho}}|w(t)|^{2} d x\right)^{2 / N}
$$

The choices of (3.4) and inequality (3.5) will be employed in the proof of (2.10), whereas (3.6) and (3.7) will be used in proving (2.11).

3-(ii). Integral estimates for $u$. Multiply (3.1) by

$$
\left(u-k_{n}\right)_{+}^{p-1} \zeta_{n}^{p}=\max \left\{0 ;\left(u-k_{n}\right)\right\}^{p-1} \zeta_{n}^{p},
$$

and integrate over $Q_{n}$ to obtain

(a)

$$
\begin{gathered}
\int_{T_{n}}^{t} \int_{B_{n}} u_{t}\left(u-k_{n}\right)_{+}^{p-1} \zeta_{n}^{p} d x d \tau \geq \frac{1}{p} \int_{\bar{B}_{n}(t)}\left(u-k_{n}\right)_{+}^{p} d x \\
-\iint_{Q_{n}}\left(u-k_{n}\right)_{+}^{p} \zeta_{n_{t}} d x d \tau, \quad T_{n+1} \leq t \leq t ;
\end{gathered}
$$

(b)

$$
\begin{aligned}
& \iint_{Q_{n}}|D u|^{p-2} D u\left\{(p-1)\left(u-k_{n}\right)_{+}^{p-2} D\left(u-k_{n}\right)_{+} \zeta_{n}^{p}\right. \\
&\left.+p\left(u-k_{n}\right)_{+}^{p-1} \zeta_{n}^{p-1} D \zeta_{n}\right\} d x d \tau \\
& \geq \frac{(p-1)}{2}\left(\frac{p}{2(p-1)}\right)^{p} \iint_{\bar{Q}_{n}}\left|D\left(u-k_{n}\right)^{2(p-1) / p}\right|^{p} d x d \tau \\
& \quad-\gamma \iint_{Q_{n}}\left(u-k_{n}\right)_{+}^{2(p-1)}\left|D \zeta_{n}\right|^{p} d x d \tau .
\end{aligned}
$$

Combining (a), (b) using (3.3) and setting

$$
w_{n}=\left(u-k_{n}\right)_{+}^{2(p-1) / p},
$$

we have

$$
\left(u-k_{n}\right)_{+}^{p}=w_{n}^{p^{2} / 2(p-1)}=w_{n}^{s} \quad(\operatorname{see}(3.4))
$$

and

$$
\begin{aligned}
& \sup _{T_{n+1} \leq t \leq T} \int_{\bar{B}_{n}(t)} w_{n}^{s} d x+\iint_{\bar{Q}_{n}}\left|D w_{n}\right|^{p} d x d \tau \\
& \quad \leq \frac{\gamma 2^{n p}}{\rho^{p}} \iint_{Q_{n}}\left(u-k_{n}\right)_{+}^{p-2}\left(u-k_{n}\right)_{+}^{p} d x d \tau+\frac{\gamma 2^{n}}{T} \iint_{Q_{n}}\left(u-k_{n}\right)_{+}^{p} d x d \tau .
\end{aligned}
$$

Define

$$
\phi(t) \equiv \phi_{r}(t) \equiv \sup _{\tau \in(0, t)} \tau^{N / \kappa} \sup _{\rho \geq r} \frac{\|u(\cdot, \tau)\|_{\infty, B_{\rho}}}{\rho^{p / p-2}},
$$

for $r>0$ fixed and $\kappa=N(p-2)+p$. Since $u_{0} \in C_{0}^{\infty}\left(\mathbf{R}^{N}\right), u \in L^{\infty}\left(\mathbf{R}^{N}\right)$ and $\phi(\cdot)$ is well defined. 
For $(x, t) \in Q_{n}$, we have $T / 4 \leq T_{n}<t<T$, and

$$
\left(u(x, t)-k_{n}\right)_{+}^{p-2} \rho^{-p} \leq\left(\frac{\|u(\cdot, t)\|_{\infty, B_{\rho}}}{\rho^{p / p-2}}\right)^{p-2} \leq \gamma t^{-N(p-2) / \kappa} \phi^{p-2}(t) .
$$

Therefore (3.9) can be written more concisely as

$$
\begin{gathered}
\sup _{T_{n+1} \leq t \leq T} \int_{\bar{B}_{n}(t)} w_{n}^{s} d x+\iint_{\bar{Q}_{n}}\left|D w_{n}\right|^{p} d x d \tau \leq \gamma 2^{n p} K(T) \iint_{Q_{n}} w_{n}^{s} d x d \tau, \\
s=p^{2} / 2(p-1), n=0,1,2, \ldots, \quad \text { where } \\
K(T)=\left(T^{-N(p-2) / \kappa} \phi^{p-2}(T)+T^{-1}\right) .
\end{gathered}
$$

3-(iii). The iteration process. From inequalities $(3.11), n=0,1,2, \ldots$, we will deduce

Lemma 3.1. There exists a constant $\gamma=\gamma(N, p)$ such that $\forall t>0$

$$
\|u(\cdot, t)\|_{\infty, B_{\rho}} \leq \gamma[K(t)]^{(N+p) / \lambda}\left(\int_{t / 4}^{t} \int_{B_{2 \rho}} u^{p} d x d \tau\right)^{p / \lambda}
$$

where

$$
\lambda=N(p-2)+p^{2} .
$$

Proof. Let $x \rightarrow \tilde{\zeta}_{n}(x)$ be a piecewise smooth cutoff function in $\bar{B}_{n}$ that equals one on $B_{n+1}$ and such that $\left|D \tilde{\zeta}_{n}\right| \leq 2^{n+2} / \rho$. Then

$$
w_{n+1} \tilde{\zeta}_{n} \in L^{\infty}\left(T_{n+1}, T ; L^{s}\left(\bar{B}_{n}\right)\right) \cap L^{p}\left(T_{n+1}, T ; W_{0}^{1, p}\left(\bar{B}_{n}\right)\right)
$$

and recalling (3.4), (3.5)

(3.14)

$$
\begin{aligned}
& \iint_{Q_{n+1}} w_{n+1}^{q} d x d \tau \leq \iint_{\bar{Q}_{n}}\left|w_{n+1} \tilde{\zeta}_{n}\right|^{q} d x d \tau \\
& \quad \leq \gamma\left\{\iint_{\bar{Q}_{n}}\left|D w_{n}\right|^{p} d x d \tau+\frac{2^{n p}}{\rho^{p}} \iint_{\bar{Q}_{n}} w_{n}^{p} d x d \tau\right\}\left(\sup _{T_{n+1} \leq t \leq T} \int_{\bar{B}_{n}(t)} w_{n}^{s} d x\right)^{\frac{p}{N}},
\end{aligned}
$$

where we have used the fact that $w_{n+1} \leq w_{n}, \forall n \in \mathbf{N}$.

From the definition (3.8) of $w_{n}$ and the definition (3.10) of $t \rightarrow \phi(t)$ it follows that

$$
\rho^{-p} \iint_{\bar{Q}_{n}} w_{n}^{p} d x d \tau \leq \gamma K(T) \iint_{Q_{n}} w_{n}^{s} d x d \tau .
$$

Substitute this in (3.14) and estimate the right-hand side by making use of (3.11) to obtain

$$
\iint_{Q_{n+1}} w_{n+1}^{q} d x d \tau \leq \gamma\left[2^{n p} K(T)\right]^{(N+p) / N}\left(\iint_{Q_{n}} w_{n}^{s} d x d \tau\right)^{(N+p) / N}
$$


By Hölder inequality and the definition of $w_{n}$

where

$$
\iint_{Q_{n+1}}\left(u-k_{n+1}\right)_{+}^{p} d x d \tau \leq\left(\iint_{Q_{n+1}} w_{n}^{q} d x d \tau\right)^{s / q}\left|A_{n+1}\right|^{1-s / q},
$$

$$
A_{n} \equiv\left\{(x, t) \in Q_{n} \mid u(x, t)>k_{n}\right\}, \quad n=0,1,2, \ldots,
$$

and $\left|A_{n}\right|=$ meas $A_{n}$. Since

$$
\begin{aligned}
\iint_{Q_{n}}\left(u-k_{n}\right)_{+}^{p} d x d \tau & \geq \iint_{Q_{n} \cap\left[u>k_{n+1}\right]}\left(u-k_{n}\right)_{+}^{p} d x d \tau \\
& \geq\left|k_{n+1}-k_{n}\right|^{p}\left|A_{n+1}\right|=\frac{k^{p}}{2^{(n+1) p}}\left|A_{n+1}\right|,
\end{aligned}
$$

we deduce from (3.15)

$$
\begin{gathered}
\iint_{Q_{n+1}}\left(u-k_{n+1}\right)_{+}^{p} d x d \tau \leq \gamma b^{n}[K(T)]^{\frac{N+p}{N} \frac{s}{q}} k^{-p\left(1-\frac{s}{q}\right)} \cdot\left(\iint_{Q_{n}}\left(u-k_{n}\right)_{+}^{p} d x d \tau\right)^{1+\frac{p}{\alpha} q}, \\
b=\left(2^{p}\right)^{1+\frac{p}{N} \frac{s}{q}} .
\end{gathered}
$$

It follows from Lemma 5.6 of $[13$, p. 95] that

provided

$$
\iint_{Q_{n}}\left(u-k_{n}\right)_{+}^{p} d x d \tau \rightarrow \iint_{Q_{\infty}}(u-k)_{+}^{p} d x d \tau=0
$$

$$
\iint_{Q_{0}} u^{p} d x d \tau \leq \gamma[K(T)]^{-(N+p) / N} k^{N\left(\frac{q}{s}-1\right)}
$$

where $Q_{\infty} \equiv B_{\rho} \times(T / 2, T), Q_{0} \equiv B_{2 \rho} \times(T / 4, T)$, and $\gamma=\gamma(N, p)$.

Using (3.4) a quick calculation shows that if $k$ is chosen to satisfy

$$
k=\gamma[K(T)]^{(N+p) / \lambda}\left(\iint_{Q_{0}} u^{p} d x d \tau\right)^{p / \lambda}, \quad \lambda=N(p-2)+p^{2},
$$

then $\sup _{Q_{\infty}} u \leq k$. Since $T>0$ is arbitrary, the lemma follows.

3-(iv). The main estimates. Define

$$
\psi(t)=\sup _{\tau \in(0, t)}\|u(\cdot, \tau)\|_{r}, \quad r>0 .
$$

Proposition 3.1. There exist positive constants $\gamma_{i}=\gamma_{i}(N, p), i=0,1,2$, such that $\forall r>0$

(3.18) $\phi(t) \leq \gamma_{1}\left\|u_{0}\right\|_{r}^{p / \kappa}, \quad \kappa=N(p-2)+p, \phi$ defined in (3.10),

(3.19) $\psi(t) \leq \gamma_{2} \mid\left\|u_{0}\right\|_{r}$,

for all $t$ satisfying

$$
0<t<\gamma_{0}\left\|u_{0}\right\|_{r}^{-(p-2)} .
$$

In the process of proving the proposition we will also demonstrate estimates (2.12) $-(2.14)$ with \|\|$\mu\|\|_{r}$ replaced by $\left\|\mid u_{0}\right\|_{r}$.

The proof proceeds in several steps. 
Lemma 3.2. There holds, $\forall t>0$

$$
\phi(t) \leq \gamma \int_{0}^{t} \tau^{-N(p-2) / \kappa} \phi(\tau)^{p-1} d \tau+\gamma \psi(t)^{p / \lambda},
$$

where $\gamma=\gamma(N, p)$.

Proof. Divide both sides of the inequality of Lemma 3.1 by $\rho^{p /(p-2)}$ and multiply by $\tau^{N / \kappa}, \tau \in(t / 2, t)$. Recalling the definition (3.12) of $K(t)$, we have for all $\tau \in(t / 2, t), \forall t>0$

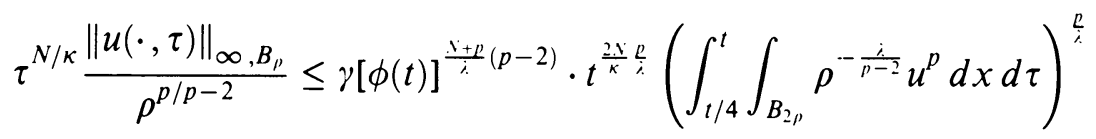

$$
\begin{aligned}
& +\gamma t^{\left(\frac{N(p-1)}{\kappa}-1\right) \frac{\rho}{i}}\left(\int_{t / 4}^{t} \int_{B_{2, \nu}} u^{p} \rho^{-\frac{i}{p-2}} d x d \tau\right) \\
& =H^{(1)}+H^{(2)} \text {. }
\end{aligned}
$$

Estimating $H^{(i)}, i=1,2$, separately we have

$$
\begin{aligned}
H^{(1)} & \leq \gamma[\phi(t)]^{1-2 p / \lambda}\left(\int_{t / 4}^{t} \tau^{-N(p-2) / \kappa}\left[\tau^{N / \kappa} \frac{\|u(\cdot, \tau)\|_{\infty, B_{2 p}}}{(2 \rho)^{p / p-2}}\right]^{p} d \tau\right)^{p / \lambda} \\
& \leq \gamma \phi(t)^{1-p / \lambda}\left(\int_{0}^{t} \tau^{-N(p-2) / \kappa} \phi^{p-1}(\tau) d \tau\right)^{p / \lambda} \\
& \leq \frac{1}{4} \phi(t)+\gamma \int_{0}^{t} \tau^{-N(p-2) / \kappa} \phi^{p-1}(\tau) d \tau \\
H^{(2)} & \leq \gamma\left\{\frac{1}{t} \int_{t / 4}^{t} \tau^{N(p-1) / \kappa}\left(\frac{\|u(\cdot, \tau)\|_{\infty, B_{2 p}}}{(2 \rho)^{p / p-2}}\right)^{p-1}(2 \rho)^{-\kappa /(p-2)} \int_{B_{2 p}} u(x, \tau) d x d \tau\right\}^{p / \lambda} \\
& \leq \gamma[\phi(t)]^{(p-1) p / \lambda}\left(\frac{1}{t} \int_{0}^{t}\|u(\cdot, \tau)\| \|_{r} d \tau\right)^{p / \lambda} \\
& \leq \gamma[\phi(t)]^{p(p-1) / \lambda}[\psi(t)]^{p / \lambda} \leq \frac{1}{4} \phi(t)+\gamma[\psi(t)]^{p / \kappa}
\end{aligned}
$$

We carry these estimates in (3.22) and take the supremum first over all $\rho \geq$ $r>0$ and then over all $\tau \in(0, t), \forall t>0$. Recalling the definition (3.10) of $t \rightarrow \phi(t)$, the lemma follows.

Lemma 3.3. Let $\rho \geq r>0$ and let $x \rightarrow \zeta(x)$ be a piecewise smooth cutoff function in $B_{2 \rho}$ such that $\zeta \equiv 1$ on $B_{\rho}$ and $|D \zeta|<\rho^{-1}$. Then $\forall t>0$

$$
\begin{aligned}
& \int_{0}^{t} \int_{B_{2, p}}|D u|^{p-1} \zeta^{p-1} d x d \tau \\
& \leq \gamma \rho \rho^{1+\kappa /(p-2)}\left(\int_{0}^{t} \tau^{(p+1) / \kappa-1} \phi(\tau)^{(p-2)(p+1) / p} \psi(\tau) d \tau\right. \\
& \left.\quad+\int_{0}^{t} \tau^{1 / \kappa-1} \phi(\tau)^{(p-2) / p} \psi(\tau) d \tau\right)^{(p-1) / p}\left(\int_{0}^{t} \tau^{1 / \kappa-1} \phi(\tau)^{(p-2) / p} \psi(\tau) d \tau\right)^{1 / p}
\end{aligned}
$$

where $\gamma=\gamma(N, p)$. 
Proof. The calculations to follow are formal in that they require $u$ to be strictly positive. They can be made rigorous by replacing $u$ with $u+\varepsilon$ and letting $\varepsilon \rightarrow 0$. By Hölder's inequality

$$
\begin{aligned}
& \int_{0}^{t} \int_{B_{2 \rho}}|D u|^{p-1} \zeta^{p-1} d x d \tau \\
&=\int_{0}^{t} \int_{B_{2 p}}\left(\tau^{(p-1) / p^{2}}|D u|^{p-1} u^{-2(p-1) / p^{2}} \zeta^{p-1}\right)\left(\tau^{-(p-1) / p^{2}} u^{2(p-1) / p^{2}}\right) d x d \tau \\
& \leq\left(\int_{0}^{t} \int_{B_{2,}} \tau^{1 / p}|D u|^{p} u^{-2 / p} \zeta^{p} d x d \tau\right)^{(p-1) / p} \\
& \\
& \cdot\left(\int_{0}^{t} \int_{B_{2 \rho}} \tau^{-(p-1) / p} u^{2(p-1) / p} d x d \tau\right)^{1 / p} \\
& \equiv {\left[J_{1}(t)\right]^{(p-1) / p}\left[J_{2}(t)\right]^{1 / p} . }
\end{aligned}
$$

To estimate $J_{1}(t)$ we multiply $(3.1)$ by $\tau^{1 / p} u^{1-2 / p} \zeta^{p}$ and integrate by parts. Standard calculations yield

$$
\begin{aligned}
& \int_{0}^{t} \int_{B_{2 \rho}} \tau^{1 / p}|D u|^{p} u^{-2 / p} \zeta^{p} d x d \tau \\
& \quad \leq \gamma \rho^{-p} \int_{0}^{t} \int_{B_{2 \rho}} \tau^{1 / p} u^{p-2 / p} d x d \tau+\gamma \int_{0}^{t} \int_{B_{2 \rho}} \tau^{1 / p-1} u^{2(p-1) / p} d x d \tau \\
& \quad \equiv L_{1}+L_{2} .
\end{aligned}
$$

Estimating $L_{i}, i=1,2$, separately, we have

$$
\begin{aligned}
L_{1} \leq & \gamma \rho^{1+\kappa /(p-2)} \int_{0}^{t} \tau^{(p+1) / \kappa-1}\left(\tau^{N / \kappa} \frac{\|u(\cdot, \tau)\|_{\infty, B_{2} p}}{(2 \rho)^{p / p-2}}\right)^{(p-2)(p+1) / p} \\
& \cdot\left(\rho^{-\kappa /(p-2)} \int_{B_{2,}} u(x, \tau) d x\right) d \tau \\
\leq & \gamma \rho^{1+\kappa /(p-2)} \int_{0}^{t} \tau^{(p+1) / \kappa-1} \phi(\tau)^{(p-2)(p+1) / p} \psi(\tau) d \tau \\
L_{2} \leq & \gamma \rho^{1+\kappa /(p-2)} \int_{0}^{t} \tau^{1 / \kappa-1}\left(\tau^{N / \kappa} \rho^{-p /(p-2)}\|u(\cdot, \tau)\|_{\infty, B_{2,}}\right)^{(p-2) / p} \\
& \cdot\left(\rho^{-\kappa /(p-2)} \int_{B_{2,}} u(x, \tau) d x\right) d \tau \\
\leq & \gamma \rho^{1+\kappa /(p-2)} \int_{0}^{t} \tau^{1 / \kappa-1} \phi(\tau)^{(p-2) / p} \psi(\tau) d \tau .
\end{aligned}
$$

On the other hand, $J_{2}(t) \equiv L_{2}$; therefore combining (3.26) and (3.25) the conclusion follows. 
Lemma 3.4. There holds

$$
\begin{aligned}
\psi(t) \leq \gamma\|\| u_{0} \|_{r}+\gamma\left(\int_{0}^{t} \tau^{(p+1) / \kappa-1} \phi(\tau)^{(p-2)(p+1) / p} \psi(\tau) d \tau\right. \\
\\
\left.+\int_{0}^{t} \tau^{1 / \kappa-1} \phi(\tau)^{(p-2) / 2} \psi(\tau) d \tau\right)
\end{aligned}
$$

where $\gamma=\gamma(N, p)$.

Proof. Let $x \rightarrow \zeta(x)$ be the cutoff function introduced in the previous lemma. Multiply (3.1) by $\zeta^{p}$ and integrate by parts to obtain after standard calculations

$$
\int_{B_{\rho}} u(x, t) d x \leq \int_{B_{2 \rho}} u_{0}(x) d x+\frac{\gamma}{\rho} \int_{0}^{t} \int_{B_{2 \rho}}|D u|^{p-1} \zeta^{p-1} d x d \tau
$$

valid for all $t>0$ and all $\rho \geq r>0$. Multiply both sides by $\rho^{-\kappa /(p-2)}$, estimate the last term by Lemma 3.3, and take the supremum over all $\rho \geq r>0$.

We are now in the position of proving Proposition 3.1 .

Lemma 3.5. Let $t \rightarrow \phi(t), \psi(t)$ be two continuous nondecreasing functions defined for $t>0$ and satisfying

$$
\begin{aligned}
\phi(t) \leq & \gamma \int_{0}^{t} \tau^{-N(p-2) / \kappa} \phi(\tau)^{p-1} d \tau+\gamma[\psi(t)]^{p / \kappa} \\
\psi(t) \leq & \gamma\|\| u_{0} \|\left.\right|_{r}+\gamma \int_{0}^{t} \tau^{(p+1) / \kappa-1} \phi(\tau)^{(p-2)(p+1) / p} \psi(\tau) d \tau \\
& +\gamma \int_{0}^{t} \tau^{1 / \kappa-1} \phi(\tau)^{(p-2) / p} \psi(\tau) d \tau
\end{aligned}
$$

for a given constant $\gamma$, and $\kappa=N(p-2)+p$. Then there exist constants $\gamma_{0}$, $\gamma_{1}, \gamma_{2}$ depending only upon $\gamma, N, p$ such that

$$
\phi(t) \leq\left.\gamma_{1}\left\|u_{0}\right\|\right|_{r} ^{p / \kappa}, \quad \psi(t) \leq \gamma_{2} \mid\left\|u_{0}\right\|_{r},
$$

for all $0<t<\gamma_{0}\left\|u_{0}\right\|_{r}^{-(p-2)}$.

Proof. First observe that $t \rightarrow \psi(t)$ being nondecreasing, $\forall t^{*}>0$ we have from (3.28a)

$$
\phi(t) \leq \gamma \int_{0}^{t} \tau^{-N(p-2) / \kappa} \phi(\tau)^{p-1} d \tau+\gamma\left[\psi\left(t^{*}\right)\right]^{p / \kappa} \quad \forall 0<t \leq t^{*} .
$$

The number $t^{*}$ will be chosen later; at this stage is an arbitrary positive value of $t$. It follows from (3.30) that $\phi(t)$ is majorized by the solution of

$$
H^{\prime}(t)=\gamma t^{-N(p-2) / \kappa} H^{p-1}(t), \quad H(0)=\gamma\left[\psi\left(t^{*}\right)\right]^{p / \kappa} .
$$

Solving explicitly

$$
\phi(t) \leq H(t)=\gamma \psi^{p / \kappa}\left(t^{*}\right)\left(1-\gamma(p-2)\left[t \psi\left(t^{*}\right)^{p-2}\right]^{p / \kappa}\right)^{-1 /(p-2)},
$$

provided the bracket is positive $\forall t \in\left[0, t^{*}\right]$. 
If this is the case the estimate above holds for $t=t^{*}$ and since $t^{*}$ is an arbitrary positive number we deduce that

$$
\phi(t) \leq \gamma \psi(t)^{p / \kappa}\left(1-\gamma(p-2)\left[t \psi(t)^{p-2}\right]^{p / \kappa}\right)^{1 /(p-2)}
$$

for all $t>0$ for which the bracket is positive. If on $t$ we impose

we obtain the statement:

$$
\gamma\left(t \psi(t)^{p-2}\right)^{p / \kappa} \leq \frac{2^{2-p}}{p-2}\left(2^{p-2}-1\right),
$$

There are two constants $\bar{\gamma}_{1}, \bar{\gamma}_{0}$ depending only on $N, p$ such that

$$
\phi(t) \leq \bar{\gamma}_{1} \psi(t)^{p / \kappa},
$$

for all $t$ satisfying

$$
\left(t \psi^{p-2}(t)\right)^{p / \kappa} \leq \bar{\gamma}_{0} .
$$

We remark that $\bar{\gamma}_{1}, \bar{\gamma}_{0}$ are quantitatively a priori determined only in dependence on $N, p$.

We now carry (3.31) into (3.28b) and obtain

$$
\psi(t) \leq \gamma\|\| u_{0} \mid \|_{r}+\gamma \int_{0}^{t} \tau^{1 / \kappa-1} \psi(\tau)^{1+(p-2) / \kappa} d \tau
$$

for all $t$ for which (3.32) holds and for a new constant $\gamma=\gamma(N, p)$.

It follows that $\psi(\cdot)$ is majorized by the solution of

$$
M^{\prime}(t)=\gamma t^{1 / \kappa-1} M(t)^{1+(p-2) / \kappa}, \quad M(0)=\gamma\left|\left\|u_{0} \mid\right\|_{r},\right.
$$

whence

$$
\psi(t) \leq M(t)=\|\| u_{0}\|\|_{r}\left(1-\gamma\left(t \mid\left\|u_{0}\right\|_{r}^{p-2}\right)^{p / \kappa}\right)^{-\kappa /(p-2)},
$$

provided the last bracket is positive.

The constant $\gamma$ being quantitatively determined a priori, we can find a constant $\overline{\bar{\gamma}}_{0}$ such that

$$
\psi(t) \leq \gamma_{2}||\left|u_{0}\right| \|_{r}
$$

as long as $t$ is so small as to satisfy (3.32) and

$$
0<t<\overline{\bar{\gamma}}_{0}\left\|u_{0}\right\|_{r}^{-(p-2)} \text {. }
$$

Putting together (3.32), (3.35), and (3.34), we deduce that there exists a constant $\gamma_{0}=\gamma_{0}(N, p)$ such that if $0<t<\gamma_{0}\left\|u_{0}\right\| \|^{-(p-2)},(3.35)$ and (3.32) hold.

Now combining (3.34) and (3.31) the lemma follows.

Corollary 3.1. There exist constants $\gamma_{0}, \gamma_{1}, \gamma_{2}$ depending only on $N, p$ such that $\forall \rho \geq r>0$

for all $0<t<\gamma_{0}\left\|u_{0}\right\|_{r}^{-(p-2)}$.

$$
\begin{aligned}
\|u(\cdot, t)\|_{\infty, B_{\rho}} & \leq \gamma_{1} \frac{\rho^{p /(p-2)}}{t^{N / \kappa}}\left\|u_{0}\right\| \|_{r}^{p / \kappa}, \\
\|u(\cdot, t)\|_{r} & \leq \gamma_{2}\left\|u_{0}\right\|_{r}
\end{aligned}
$$


As a by-product we obtain estimate (2.14), i.e.,

Corollary 3.2. There exist constants $\gamma, \gamma_{0}=\gamma, \gamma_{0}(N, p)$ such that $\forall \rho \geq r>0$,

$$
\int_{0}^{t} \int_{B_{\rho}}|D u|^{p-1} d x d \tau \leq \gamma t^{1 / \kappa} \rho^{1+\kappa /(p-2)} \mid\left\|u_{0}\right\|_{r}^{1+(p-2) / \kappa}
$$

$\forall 0<t<\gamma_{0}\|\| u_{0} \|_{r}^{-(p-2)}$.

Estimate (2.12) follows from a technique similar to that of Lemma 3.3.

For simplicity let us assume $\Omega \equiv B_{\rho}$ and let $Q \equiv B_{\rho} \times(0, T)$ where $\rho \geq$ $r>0$ and

$$
T \leq C_{0}\left\|u_{0}\right\|_{r}^{-(p-2)}
$$

and $C_{0}$ is the constant claimed by $(2.8)$ of Theorem 1 and previously found in a quantitative way.

Setting

$$
q=p-(N+\varepsilon) /(N+1), \quad \varepsilon>0,
$$

we have $\forall \alpha, \beta \in \mathbf{R}^{+}$, formally

$$
\begin{aligned}
\iint_{Q}|D u|^{q} d x d \tau=\iint_{Q} t^{\beta} u^{-\alpha}|D u|^{q} t^{-\beta} u^{\alpha} d x d t \\
\quad \leq\left(\iint_{Q} t^{\beta p / q} \frac{|D u|^{p}}{u^{\alpha p / q}} d x d \tau\right)^{q / p}\left(\iint_{Q} t^{-\beta p /(p-q)} u^{\alpha p /(p-q)} d x d \tau\right)^{1-q / p} \\
\quad=\left(I^{(1)}\right)^{q / p}\left(I^{(2)}\right)^{1-q / p}
\end{aligned}
$$

Multiply (3.1) by $t^{\beta p / q} u^{1-\alpha p / q} \zeta(x)$, with the choice $\alpha p=p-q$ so that

$$
1-\alpha p / q=2-p / q=(p-2(N+\varepsilon) /(N+1)) / q .
$$

Here $x \rightarrow \zeta(x)$ is the usual cutoff function in $B_{2 \rho}$. By standard calculations we find

$$
\begin{aligned}
I^{(1)} \leq & \frac{\gamma}{\rho^{p}} \iint_{Q_{2 \rho}} t^{\beta \frac{p}{q}} u^{p-2+\left(1-\alpha_{q}^{\left.\frac{p}{q}\right)}\right.} u d x d t \\
& +\gamma \iint_{Q_{2 \rho}} t^{\beta \frac{p}{q}-1} u^{1-\alpha_{q}^{p}} u d x d t .
\end{aligned}
$$

To estimate the first integral on the right-hand side of (3.37) we use Corollary 3.1 to conclude

$$
\frac{\gamma}{\rho^{p}} \iint_{Q_{2 \rho}} t^{\beta \frac{p}{q}} u^{p-2} u^{\left(1-\alpha_{q}^{p}\right)} u d x d t \leq \gamma\left(\rho,\left\|\mid u_{0}\right\|_{r}\right)\left(\int_{0}^{t} t^{\beta \frac{p}{q}-N / \kappa\left(1-\alpha_{q}^{p}\right)-1} d t\right) .
$$

The second integral in (3.37) behaves analogously and we obtain

provided

$$
I^{(1)} \leq \gamma\left(\rho,\|\| u_{0}\|\|_{r}\right) T^{\beta_{q}^{p}-\frac{N}{\kappa}\left(1-\alpha_{q}^{p}\right)},
$$

$$
\beta \frac{p}{q}-\frac{N}{\kappa}\left(1-\alpha \frac{p}{q}\right)>0 .
$$


As for $I^{(2)}$ we have

$$
I^{(2)} \leq \gamma\left(\rho,\left\|\left|u_{0}\right|\right\|_{r}\right) T^{1-\beta p /(p-q)},
$$

provided

$$
1-\beta p /(p-q)>0
$$

Condition (3.38) yields

$$
\beta p>\frac{N}{\kappa}\left(p-\frac{2(N+\varepsilon)}{N+1}\right)
$$

and (3.39) gives $\beta<(N+3) /(N+1)$.

A quick calculation shows that these conditions are compatible only if $\varepsilon>0$.

\section{Proof of Theorem 1. The gradient estimate}

Take the $x_{i}$-derivative in (3.1) to obtain formally

$$
\frac{\partial}{\partial t} u_{x_{i}}=\operatorname{div}\left\{|D u|^{p-2} D u_{x_{i}}+(p-2)|D u|^{p-3} \frac{\partial}{\partial x_{i}}|D u| D u\right\}=0
$$

Multiply this by the testing functions

$$
\begin{aligned}
\eta_{n} & =2 u_{x_{i}}(v-k)_{+}^{\alpha} \zeta_{n}^{2}, \quad \alpha>0 \\
v & =|D u|^{2}
\end{aligned}
$$

and integrate over $Q_{n}$. Here $\zeta_{n}$ and $Q_{n}$ are as in $\S 3-(i)$. Proceeding formally, we have (repeated indices denote summation over those indices)

(a) $2 \int_{T_{n}}^{t} \int_{B_{n}} \frac{\partial}{\partial t} u_{x_{i}} u_{x_{i}}(v-k)_{+}^{\alpha} \zeta_{n}^{2} d x d \tau$

$$
\begin{aligned}
& =\frac{1}{\alpha+1} \int_{T_{n}}^{t} \int_{B_{n}} \frac{\partial}{\partial t}(v-k)_{+}^{\alpha+1} \zeta_{n}^{2} d x d \tau \\
& \geq \frac{1}{\alpha+1} \int_{\bar{B}_{n}(t)}(v-k)_{+}^{\alpha+1} d x-\frac{2}{\alpha+1} \iint_{Q_{n}}(v-k)_{+}^{\alpha+1} \zeta_{n} \zeta_{n_{t}} d x d \tau,
\end{aligned}
$$

$$
\forall T_{n+1}<t<T .
$$




$$
\begin{aligned}
& \text { (b) }-\iint_{Q_{n}} \operatorname{div}\left\{|D u|^{p-2} D u_{x_{i}}+(p-2)|D u|^{p-3} \frac{\partial}{\partial x_{i}}|D u| \cdot D u\right\} \eta_{n} d x d \tau \\
&=\iint_{Q_{n} \cap[v>k]}|D u|^{p-2}\left\{\alpha|D v|^{2}(v-k)_{+}^{\alpha-1} \zeta_{n}^{2}\right. \\
& \quad+2 \sum_{i=1}^{N}\left|D u_{x_{i}}\right|^{2}(v-k)_{+}^{\alpha} \zeta_{n}^{2} \\
& \quad+\left.(p-2) \iint_{Q_{n}}|D u|^{p-2} \sum_{i=1}^{N}\left|\frac{\partial}{\partial x_{i}}\right| D u\right|^{2}(v-k)_{+}^{\alpha} \zeta_{n}^{2} d x d \tau \\
& \quad+2 \alpha(p-2) \iint_{Q_{n} \cap[v>k]}|D u|^{p-2}|D(|D u|) \cdot D u|^{2}(v-k)_{+}^{\alpha-1} \zeta_{n}^{2} d x d \tau \\
& \quad+4(p-2) \iint_{Q_{n}}|D u|^{p-3}(D(|D u|) \cdot D u)(v-k)_{+}^{\alpha} D u \zeta_{n} D \zeta_{n} d x d \tau \\
& \geq \frac{4 \alpha}{(\alpha+1)^{2}} \iint_{\bar{Q}_{n}}|D u|^{p-2}\left|D(v-k)^{(\alpha+1) / 2}\right|^{2} d x d \tau \\
&-\frac{\gamma 2^{2 n}}{\alpha \rho^{2}} \iint_{Q_{n}}|D u|^{p-2}(v-k)_{+}^{\alpha+1} d x d \tau .
\end{aligned}
$$

Combining (a) and (b) yields

$$
\begin{aligned}
& \sup _{T_{n+1} \leq t \leq T} \int_{\bar{B}_{n}(t)}(v-k)_{+}^{\alpha+1} d x+\iint_{\bar{Q}_{n}}|D u|^{p-2}\left|D(v-k)^{(\alpha+1) / 2}\right|^{2} d x d \tau \\
& \quad \leq \frac{\gamma 2^{2 n}}{\rho^{2}} \iint_{Q_{n}}|D u|^{p-2}(v-k)_{+}^{\alpha+1} d x d \tau+\frac{\gamma 2^{2 n}}{T} \iint_{Q_{n}}(v-k)_{+}^{\alpha+1} d x d \tau,
\end{aligned}
$$

where $\gamma=\gamma(N, p, \alpha)$.

These calculations are somewhat formal. They can be rigorously justified by first writing (4.1) in terms of difference quotients in the space variables and Steklov averagings in $t$. Then in the weak formulation one takes test functions $\eta_{n}$ where the derivatives appear as difference quotients and further they are averaged in time. A standard limiting process makes the result rigorous. Also, it is easily seen that the integrals involving $(v-k)_{+}^{\alpha-1}(\alpha>0)$ are well defined even if $\alpha-1<0$.

Let $\left\{k_{n}\right\}$ be the increasing sequence

$$
k_{n}=k-k / 2^{n+1}, \quad n=0,1,2, \ldots,
$$

where $k>0$ will be chosen later. Define

$$
A_{n} \equiv\left\{(x, t) \in Q_{n} \mid v(x, t)>k_{n}\right\} ; \quad v=|D u|^{2},
$$


and observe that on the set $A_{n}$

$$
|D u|^{p-2} \geq\left(\frac{1}{2} k\right)^{(p-2) / 2}
$$

Also define

$$
\Phi(t)=\sup _{\tau \in(0, t)} \tau^{(N+1) / \kappa} \sup _{\substack{\rho \geq r>0 \\ \kappa=N(p-2)+p .}} \frac{\|D u\|_{\infty, B_{\rho}}(\tau)}{\rho^{2 /(p-2)}}, \quad p>2,
$$

In view of (2.10) and the results of [9], it is easily seen that $\Phi(t)$ is well defined, by possibly working in $\mathbf{R}^{N} \times(\varepsilon, \infty)$ with $\varepsilon \in(0,1)$ arbitrary and then letting $\varepsilon \rightarrow 0$. Then for all $t \in(T / 2, T)$

$$
|D u|^{p-2} / \rho^{2} \leq \gamma T^{-(N+1)(p-2) / \kappa} \Phi(t)^{p-2} \text {. }
$$

We write (4.3) with $k$ replaced by $k_{n}$ and set

$$
w_{n}=\left(v-k_{n}\right)^{(\alpha+1) / 2}, \quad \alpha>0 \text {. }
$$

Then carrying the remarks above into (4.3) we have in a more concise way

$$
\begin{aligned}
& \sup _{T_{n+1} \leq t \leq T} \int_{\bar{B}_{n}(t)} w_{n}^{2} d x+k^{(p-2) / 2} \iint_{\bar{Q}_{n}}\left|D w_{n}\right|^{2} d x d \tau \\
& \quad \leq \gamma 2^{2 n} H(T) \iint_{Q_{n}} w_{n}^{2} d x d \tau,
\end{aligned}
$$

where

$$
H(T) \equiv\left[T^{((N+1) / \kappa)(p-2)} \Phi^{p-2}(T)+T^{-1}\right] .
$$

Let $x \rightarrow \tilde{\zeta}_{n}(x)$ be a cutoff function in $\bar{B}_{n}$ which equals one on $B_{n+1}$ and such that $\left|D \tilde{\zeta}_{n}\right| \leq 2^{n} / \rho$. Then

$$
w_{n} \tilde{\zeta}_{n} \in L^{\infty}\left(T_{n+1}, T ; L^{2}\left(\bar{B}_{n}\right)\right) \cap L^{2}\left(T_{n+1}, T ; W_{0}^{1,2}\left(\bar{B}_{n}\right)\right) \text {, }
$$

and applying (3.7)

$$
\begin{aligned}
\iint_{Q_{n+1}} w_{n}^{2(N+2) / N} d x d \tau \leq & \iint_{\bar{Q}_{n}}\left(w_{n} \tilde{\zeta}_{n}\right)^{2(N+2) / N} d x d \tau \\
\leq & \left.\gamma \iiint_{\bar{Q}_{n}}\left|D w_{n}\right|^{2} d x d \tau+\frac{2^{2 n}}{\rho^{2}} \iint_{\bar{Q}_{n}} w_{n}^{2} d x d \tau\right\} \\
& \cdot\left(\sup _{T_{n+1} \leq t \leq T} \int_{\bar{B}_{n}(t)} w_{n}^{2} d x\right)^{2 / N} .
\end{aligned}
$$

Next we impose on $k$ the restriction

$$
0<k<\rho^{4 /(p-2)} T^{-2(N+1) / \kappa} \Phi^{2}(T) .
$$

Then

$$
\begin{aligned}
\frac{2^{2 n}}{\rho^{2}} \iint_{\bar{Q}_{n}} w_{n}^{2} d x d \tau & \leq \frac{2^{2 n}}{\rho^{2}} \frac{k^{(p-2) / 2}}{k^{(p-2) / 2}} \iint_{\bar{Q}_{n}} w_{n}^{2} d x d \tau \\
& \leq \gamma 2^{2 n} \frac{H(T)}{k^{(p-2) / 2}} \iint_{Q_{n}} w_{n}^{2} d x d \tau
\end{aligned}
$$


We substitute this in (4.9) and estimate the right-hand side by making use of (4.7) to obtain

$$
\begin{aligned}
& \iint_{Q_{n+1}} w_{n}^{2(N+2) / N} d x d \tau \\
& \quad \leq \gamma\left[2^{2 n} H(T)\right]^{(N+2) / N} k^{-(p-2) / 2}\left(\iint_{Q_{n}} w_{n}^{2} d x d \tau\right)^{(N+2) / N}
\end{aligned}
$$

By Hölder inequality

$$
\iint_{Q_{n+1}} w_{n}^{2} d x d \tau \leq\left(\iint_{Q_{n+1}} w_{n}^{2(N+2) / N} d x d \tau\right)^{N /(N+2)}\left|A_{n+1}\right|^{2 /(N+2)}
$$

and since $\left|A_{n+1}\right| \leq 2^{(\alpha+1)(n+1)} k^{-(\alpha+1)} \iint_{Q_{n}}\left(v-k_{n}\right)_{+}^{\alpha+1} d x d \tau$, we obtain from (4.11) and (4.12)

$$
\begin{gathered}
\iint_{Q_{n+1}}\left(v-k_{n+1}\right)_{+}^{\alpha+1} d x d \tau \leq \gamma b^{n} H(T) k^{-(N(p-2)+4(\alpha+1)) / 2(N+2)} \\
\cdot\left(\iint_{Q_{n}}\left(v-k_{n}\right)_{+}^{\alpha+1} d x d \tau\right)^{1+2 /(N+2)}, \\
b=2^{2+(\alpha+1) \frac{2}{N+2}}, \quad \gamma=\gamma(N, p, \alpha) .
\end{gathered}
$$

It then follows from Lemma 5.6 of $[13, \mathrm{p} .95]$ that

$$
\iint_{Q_{n}}\left(v-k_{n}\right)_{+}^{\alpha+1} d x d \tau \rightarrow 0 \text { as } n \rightarrow \infty
$$

provided

$$
\iint_{Q_{0}}\left(v-\frac{k}{2}\right)_{+}^{\alpha+1} d x d \tau \leq \gamma[H(T)]^{-(N+2) / 2} k^{(N(p-2)+4(\alpha+1)) / 4} .
$$

This condition is satisfied if we choose

$$
\begin{gathered}
k=\gamma H(T)^{2(N+2) / \sigma}\left(\int_{T / 4}^{T} \int_{B_{2 p}}|D u|^{2(\alpha+1)} d x d \tau\right)^{4 / \sigma} \\
\sigma=N(p-2)+4(\alpha+1) .
\end{gathered}
$$

If the choice of (4.13) is compatible with (4.10), then $\forall t \in(T / 2, T)$

$$
\|D u\|_{\infty, B_{\rho}}(t) \leq \gamma H(T)^{(N+2) / \sigma}\left(\int_{T / 4}^{T} \int_{B_{2, \nu}}|D u|^{2(\alpha+1)} d x d \tau\right)^{2 / \sigma} .
$$

If on the other hand (4.13) is not compatible with (4.10), we have

$$
\rho^{2 /(p-2)} T^{-(N+1) / \kappa} \Phi(T) \leq \gamma H(T)^{(N+2) / \sigma}\left(\int_{T / 2}^{T} \int_{B_{2, \nu}}|D u|^{2(\alpha+1)} d x d t\right)^{2 / \sigma}
$$

and recalling the definition (4.5) of $\Phi(T)$, this again implies (4.15). Hence (4.15) holds in either case by suitably modifying the constant $\gamma$. 
To proceed we choose $\alpha=(p-2) / 2$ so that

$$
\sigma=N(p-2)+2 p .
$$

Divide both sides of (4.15) by $\rho^{2 /(p-2)}$ and multiply by $t^{(N+1) / \kappa}$. Then $\forall t>0$, recalling the definition (4.8) of $H(t)$, we have

$$
\begin{aligned}
t^{(N+1) / \kappa} \frac{\|D u\|_{\infty, B_{\rho}}(t)}{\rho^{2 / p-2}} \leq & \gamma \Phi(t)^{\frac{N+2}{\sigma}(p-2)} t^{\frac{N+1}{\kappa} \frac{4}{\sigma}} \cdot\left(\int_{t / 4}^{t} \rho^{-\frac{\sigma}{p-2}} \int_{B_{2 \rho}}|D u|^{p} d x d \tau\right)^{2 / \sigma} \\
& +\gamma\left(t^{N / \kappa} \int_{t / 4}^{t} \rho^{-\frac{\sigma}{p-2}} \int_{B_{2 \rho}}|D u|^{p} d x d \tau\right)^{2 / \sigma} \equiv G^{(1)}+G^{(2)} .
\end{aligned}
$$

Estimating $G^{(1)}$ we have

$$
\begin{aligned}
G^{(1)} & \leq \gamma \Phi(t)^{\frac{(N+2)(p-2)}{\sigma}}\left\{\int_{t / 4}^{t} \tau^{-\frac{(N+1)}{\kappa}(p-2)} \tau^{\frac{(N+1)}{\kappa} p} \cdot\left(\sup _{\rho \geq r} \frac{\|D u\|_{\infty, B_{\rho}}(\tau)}{\rho^{2 / p-2}}\right)^{p} d \tau\right\}^{2 / \sigma} \\
& \leq \gamma \Phi(t)^{\frac{(N+2)(p-2)}{\sigma}+\frac{2}{\sigma}}\left(\int_{0}^{t} \tau^{-\frac{(N+1)}{\kappa}(p-2)} \Phi(\tau)^{p-1} d \tau\right)^{2 / \sigma} \\
& \leq \frac{1}{4} \Phi(t)+\gamma \int_{0}^{t} \tau^{-\frac{(N+1)}{\kappa}(p-2)} \Phi(\tau)^{p-1} d \tau .
\end{aligned}
$$

In order to estimate $G^{(2)}$ we refer back to equation (3.1). Let $(x, t) \rightarrow \zeta(x, t)$ be a piecewise smooth cutoff function in $B_{4 \rho} \times(t / 8, t)$ which equals one on $B_{2 \rho} \times(t / 4, t)$ and such that $|D \zeta| \leq 4 / \rho, 0 \leq \zeta_{t} \leq 8 / 3 t$. Multiplying (3.1) by $u \zeta^{p}$ and integrating by parts we obtain after standard calculations

$$
\int_{t / 4}^{t} \int_{B_{2 \rho}}|D u|^{p} d x d \tau \leq \frac{\gamma}{\rho^{p}} \int_{t / 8}^{t} u^{p} d x d \tau+\frac{\gamma}{t} \int_{t / 8}^{t} \int_{B_{4 \rho}} u^{2} d x d \tau .
$$

Therefore

$$
\begin{aligned}
G^{(2)} \leq & \gamma\left\{t^{N / \kappa} \sup _{p \geq r} \int_{t / 4}^{t} \rho^{-\sigma /(p-2)} \int_{B_{2 \rho}}|D u|^{p} d x d \tau\right\}^{2 / \sigma} \\
\leq & \gamma\left\{\int_{t / 8}^{t} \tau^{N / \kappa}\left(\sup _{\rho \geq r} \frac{\|u(\tau)\|_{\infty, B_{\rho}}}{\rho^{p /(p-2)}}\right)^{p-1}\left(\sup _{\rho \geq r} \rho^{-\kappa /(p-2)} \int_{B_{\rho}} u(x, \tau) d x\right) d \tau\right\}^{2 / \sigma} \\
& +\gamma\left\{\int_{t / 8}^{t} \tau^{N / \kappa-1}\left(\sup _{\rho \geq r} \frac{\|u(\tau)\|_{\infty, B_{\rho}}}{\rho^{p /(p-2)}}\right)\left(\sup _{\rho \geq r} \rho^{-\kappa /(p-2)} \int_{B_{\rho}} u(x, \tau) d x\right) d \tau\right\}^{2 / \sigma} \\
\leq & \gamma\left\{\int_{t / 8}^{t} \tau^{-(N / \kappa)(p-2)} \phi(\tau)^{p-1}\|u(\cdot, \tau) \mid\|_{r} d \tau\right\}^{2 / \sigma} \\
& +\gamma\left\{\int_{t / 8}^{t} t^{-1} \phi(\tau)\|u(\cdot, \tau) \mid\|_{r} d \tau\right\}^{2 / \sigma},
\end{aligned}
$$


where we have employed the definitions (3.10) and (2.6).

Next, applying Corollary 3.1 and the definition (3.17) of $t \rightarrow \psi(t)$,

$$
G^{(2)} \leq \gamma\left[t^{p / \kappa} \Phi(t)^{p-2}\right]^{2 / \sigma}\left[\psi(t)^{1+p / \kappa}\right]^{2 / \sigma}+\gamma\left[\psi^{1+p / \kappa}\right]^{2 / \sigma},
$$

for all $t$ within the range claimed by Corollary 3.1. Within such a range

$$
t^{p / \kappa} \phi(t)^{p-2} \leq \gamma=\gamma(N, p) \text { and } \psi(t) \leq \gamma_{2}\left\|u_{0}\right\|_{r} .
$$

It follows that

$$
G^{(2)} \leq \gamma\|\| u_{0} \|_{r}^{2 / \kappa}, \quad \gamma=\gamma(N, p)
$$

$\forall 0<t<\gamma_{0}\|\| u_{0} \|_{r}^{-(p-2)}$, where $\gamma_{0}=\gamma_{0}(N, p)$ is the number claimed by Corollary 3.1 .

We substitute these estimates in (4.17) and take the supremum first over all $\rho \geq r$ and then over all $0<t<\gamma_{0}\left\|u_{0}\right\|^{-(p-2)}$. Recalling the definition (4.5) of $t \rightarrow \Phi(t)$ we obtain

$$
\begin{gathered}
\Phi(t) \leq \int_{0}^{t} \tau^{-((N+1) / \kappa)(p-2)} \Phi(\tau)^{p-1} d \tau+\gamma\|\| u_{0} \|_{r}^{2 / \kappa}, \\
\forall 0<t<\gamma_{0}\left\|u_{0}\right\| \|_{r}^{-(p-2)} .
\end{gathered}
$$

It follows that $\Phi(\cdot)$ is majorized by the solution of

$$
\begin{aligned}
& V^{\prime}(t)=\gamma t^{-((N+1) / \kappa)(p-2)} V^{p-1}(t), \\
& V(0)=\gamma\|\| u_{0}\left\|_{r}^{2 / \kappa}, \quad 0<t<\gamma_{0}\right\| u_{0} \|_{r}^{-(p-2)} .
\end{aligned}
$$

Solving explicitly

$$
\Phi(t) \leq \gamma\left|\left\|u_{0} \mid\right\|_{r}^{2 / \kappa}\left\{1-\gamma\left(t \mid\left\|u_{0}\right\|_{r}^{p-2}\right)^{2 / \kappa}\right\}^{-1 /(p-2)},\right.
$$

and therefore if $t$ is so small that

$$
\left\{1-\gamma\left(t \mid\left\|u_{0}\right\| \|_{r}^{p-2}\right)^{2 / \kappa}\right\}^{-1 /(p-2)} \leq 2
$$

we will have

$$
t^{(N+1) / \kappa}\|D u\|_{\infty, B_{\rho}}(t) / \rho^{2 /(p-2)} \leq 2 \gamma\|\| u_{0}\|\|_{r}^{2 / \kappa},
$$

for all such $t$ and $\forall \rho \geq r$.

The gradient estimate $(2.11)$ follows readily and Theorem 1 is proved.

\section{HARNACK INEQUALITY AND INITIAL TRACES}

We will prove in this section the Harnack inequality of Theorem 3 and Lemma 2.2. The existence of initial traces and their uniqueness (Theorem 2) will follow readily.

5-(i). Harnack inequality: a special case. Let $u \geq 0$ be the unique solution of

$$
\begin{gathered}
u_{t}-\operatorname{div}\left(|D u|^{p-2} D u\right)=0 \quad \text { in } \mathbf{R}^{N} \times(0, \infty), \\
u(x, 0)=u_{0}(x) \geq 0,
\end{gathered}
$$


where we assume

$$
\left\{\begin{array}{l}
x \rightarrow u_{0}(x) \in L^{\infty}\left(B_{R}\right) \text { for some } R>0 \text { fixed } \\
\text { and } u_{0}(x)=0, \quad|x|>R
\end{array}\right.
$$

We will show that $\forall T>0$

$$
f_{B_{R}} u_{0}(x) d x \leq \gamma\left\{\left(\frac{R^{p}}{T}\right)^{1 /(p-2)}+\left(\frac{T}{R^{p}}\right)^{N / p}[u(0, T)]^{\kappa / p}\right\}
$$

for a constant $\gamma$ depending only on $N, p$ and independent of $R, \theta, u, u_{0}$. As a starting point we record the following consequence of Corollary 3.2.

Lemma 5.1. There exist constants $\gamma, \gamma_{0}=\gamma, \gamma_{0}(N, p)$ such that $\forall \rho \geq R$

$$
\int_{0}^{t} \int_{B_{\rho}}|D u|^{p-1} d x d \tau \leq \gamma t^{1 / \kappa} \rho^{1+\kappa /(p-2)}\left(R^{-p /(p-2)} f_{B_{R}} u_{0}(x) d x\right)^{1+(p-2) / \kappa}
$$

for all $t$ satisfying

$$
0<t<\gamma_{0}\left(R^{-p /(p-2)} f_{B_{R}} u_{0}(x) d x\right)^{-(p-2)} .
$$

Proof. Observe that since $x \rightarrow u_{0}(x)$ is supported in $B_{R}$

$$
\left\|u_{0}\right\| \|_{R}=\sup _{\rho \geq R} \rho^{-\kappa /(p-2)} \int_{B_{\rho}} u_{0}(x) d x=R^{-p /(p-2)} f_{B_{R}} u_{0}(x) d x .
$$

To proceed, let $\varepsilon \in(0,1]$ and let $x \rightarrow \zeta(x)$ be a piecewise smooth cutoff function in $B_{(1+\varepsilon) R}$ which equals one on $B_{R}$ and such that $|D \zeta|<(\varepsilon R)^{-1}$. Multiply (5.1) by $\zeta$ and integrate by parts to obtain

$$
\int_{B_{(1+\varepsilon) R}} u(x, t) d x \geq \int_{B_{R}} u_{0}(x) d x-\frac{1}{\varepsilon R} \int_{0}^{t} \int_{B_{2 R}}|D u|^{p-1} d x d \tau
$$

valid $\forall t>0$. Set

$$
E_{0}=f_{B_{R}} u_{0}(x) d x .
$$

Then for all $t \in\left(0, \gamma_{0} R^{p} / E_{0}^{p-2}\right)$, we obtain from Lemma 5.1 with $\rho=(1+\varepsilon) R$

$$
f_{B_{(1+\varepsilon) R}} u(x, t) d x \geq(1+\varepsilon)^{-N} E_{0}-\frac{\gamma}{\varepsilon} E_{0}\left(E_{0}^{p-2} R^{-p} t\right)^{1 / \kappa}
$$

$\forall \varepsilon \in(0,1]$.

With the aid of inequality (5.7), taking $\varepsilon=1$ we deduce

Lemma 5.2. There exists a number $\delta \in(0,1), \delta=\delta(N, p)$ such that at the time level

$$
t_{0}=\delta R^{p} / E_{0}^{p-2},
$$

within the ball $B_{2 R}$, there exists at least one point $x_{0}$ such that

$$
u\left(x_{0}, t_{0}\right) \geq 2^{-(N+1)} E_{0} \text {. }
$$


Remark 5.1. Inequality (5.7) with the parameter $\varepsilon \in(0,1)$ is needed later. For the remainder of this section we will take $\varepsilon=1$.

For notational simplicity set

$$
\bar{u}=u\left(x_{0}, t_{0}\right),
$$

and consider the box

$$
Q_{0} \equiv\left\{\left|x-x_{0}\right|<\rho\right\} \times\left\{t_{0}-\frac{\bar{C}_{1} \rho^{p}}{\bar{u}^{p-2}}, t_{0}+\frac{\bar{C}_{1} \rho^{p}}{\bar{u}^{p-2}}\right\},
$$

where $\bar{C}_{1}=\bar{C}_{1}(N, p)$ is the constant of Theorem 1 of [8] (see $\S 2$-(iv) here), and

$$
\rho^{p}=\left(\delta / 2^{N+2}\right)\left(1 / \bar{C}_{1}\right) R^{p} .
$$

In view of such a choice of $\rho$ we have

$$
t_{0}-\frac{\bar{C}_{1} \rho^{p}}{\bar{u}^{p-2}} \geq \frac{\delta R^{p}}{E_{0}^{p-2}}-\frac{\bar{C}_{1}^{N+1}}{E_{0}^{p-2}} \rho^{p} \geq \frac{\delta}{2 E_{0}^{p-2}} R^{p}>0,
$$

and therefore the box $Q_{0}$ is all contained in the domain of definition of $u$. By Theorem 1 of [8]

$$
\begin{aligned}
& \bar{u} \leq \bar{C}_{0} \inf _{x \in B_{\rho}\left(x_{0}\right)} u(x, \bar{t}), \\
& \bar{t}=t_{0}+\bar{C}_{1} \rho^{p} / \bar{u}^{p-2} .
\end{aligned}
$$

Combining (5.11) and (5.9) we have

$$
u(x, \bar{t}) \geq\left(\bar{C}_{0} 2^{N+1}\right)^{-1} E_{0}, \quad \forall x \in B_{\rho}\left(x_{0}\right) .
$$

We are now in the position of proving (5.4).

Let us fix $T>0$ and let us assume that the level $\bar{t}$ found in (5.11), (5.12) is below $T / 2$, i.e.,

$$
\bar{t}=t_{0}+\bar{C}_{1} \rho^{p} / \bar{u}^{p-2}<T / 2 .
$$

Using (5.9) and (5.10) we see that this will happen if

$$
2 \delta R^{p} / T+\left(\delta / 2^{N+2}\right) R^{p} / T<E_{0}^{p-2},
$$

i.e., if

$$
E_{0}=f_{B_{R}} u_{0}(x) d x>\bar{C}_{2}(N, p)\left(\frac{R^{p}}{T}\right)^{1 /(p-2)} .
$$

If (5.14) is violated, then (5.4) holds trivially. Therefore we assume (5.14) holds so that $\bar{t}=t_{0}+\bar{C}_{1} \rho^{p} / \bar{u}^{p-2}<T / 2$ and

$$
T-\bar{t} \geq T / 2 \text {. }
$$


To proceed, consider the following explicit solution of (5.1) in $\mathbf{R}^{N} \times[\bar{t}, \infty)$.

$$
\left\{\begin{array}{l}
\mathscr{B}_{k, \rho}\left(x, t ; x_{0} \bar{t}\right)=k \rho^{N} S(t)^{-N / \kappa}\left\{1-\left(\frac{\left|x-x_{0}\right|}{S(t)^{1 / \kappa}}\right)^{p /(p-1)}\right\}_{+}^{(p-1) /(p-2)} \\
S(t)=\left(\tilde{\gamma}_{0} k^{p-2} \rho^{N(p-2)}(t-\bar{t})+\rho^{\kappa}\right), \\
\tilde{\gamma}_{0}(N, p)=\kappa(p /(p-2))^{p-1}, \quad \kappa=N(p-2)+p, \\
k=\left(\bar{C}_{1} 2^{N+1}\right)^{-1} E_{0} .
\end{array}\right.
$$

It is readily seen that $\mathscr{B}_{k, \rho}\left(x, t ; x_{0}, \bar{t}\right)$ satisfies $(5.1)$ for $t>\bar{t}, x \in \mathbf{R}^{N}$. Moreover, for $t=\bar{t}$ it vanishes outside the ball $B_{\rho}\left(x_{0}\right)$ and $\forall t>\bar{t}$ it vanishes (in a $C^{1, \alpha}$-fashion) outside the ball $\left|x-x_{0}\right|<S(t)^{1 / \kappa}$. One also verifies that

$$
\mathscr{B}_{k, \rho}\left(x, \bar{t} ; x_{0}, \bar{t}\right) \leq k,
$$

and therefore by the maximum principle

$$
u(x, t) \geq \mathscr{B}_{k, \rho}\left(x, t ; x_{0}, \bar{t}\right) \quad \forall t \geq \bar{t} .
$$

In (5.17) choose

$$
t=T, \quad x=0 .
$$

Since $x_{0} \in B_{2 R}$ we will have

$$
\left\{1-\left(\frac{\left|x_{0}\right|}{S(T)^{1 / \kappa}}\right)^{p /(p-1)}\right\}^{(p-1) /(p-2)} \geq\left(\frac{1}{2}\right)^{(p-1) /(p-2)}
$$

if $S(T) \geq 2^{(2-1 / p) \kappa} R^{\kappa}$.

Using (5.16) and (5.10) we see that this will happen if

$$
\bar{C}_{3}(N, p) E_{0}^{p-2} R^{N(p-2)} T+\bar{C}_{4}(N, p) R^{\kappa}>R^{\kappa}
$$

and this in turn will be verified if $E_{0}$ satisfies

$$
E_{0}=f_{B_{R}} u_{0}(x) d x>\bar{C}_{5}(N, p)\left(\frac{R^{p}}{T}\right)^{1 /(p-2)} .
$$

Let us assume (5.19) for the moment. Then from (5.17) with the choices (5.18) we obtain

Now if (5.19) holds

$$
u(0, T) \geq \bar{C}_{6}(N, p) E_{0} R^{N} / S(T)^{N / \kappa} .
$$

$$
\begin{aligned}
S(T) & \leq \bar{C}_{7}(N, p) E_{0}^{p-2} R^{N(p-2)} T+\bar{C}_{8}(N, p) R^{\kappa} \\
& \leq \bar{C}_{9}(N, p) E_{0}^{p-2} R^{N(p-2)} T \\
& =\bar{C}_{10}(N, p) E_{0}^{p-2}\left(T / R^{p}\right) R^{\kappa}
\end{aligned}
$$

and therefore

$$
u(0, T) \geq \bar{C}_{11}(N, p) E_{0}^{1-N(p-2) / \kappa}\left(R^{p} / T\right)^{N / \kappa} .
$$


This in turn implies

$$
f_{B_{R}} u_{0}(x) d x=E_{0} \leq \bar{C}_{12}(N, p)\left(\frac{T}{R^{p}}\right)^{N / p}[u(0, T)]^{\kappa / p} .
$$

On the other hand, if (5.19) is violated, then (5.4) holds trivially.

The comparison function $\mathscr{B}_{k, \rho}$ introduced in (5.16) will also be useful in the next section.

5-(ii). Harnack inequality: the general case. Let $u \geq 0$ be a solution of (2.1) in $S_{T}, 0<T<\infty$. Fix $R>0, \tau \in(0, T / 2]$ and let $(x, t) \rightarrow w(x, t)$ be the unique solution of

$$
\begin{gathered}
w_{t}-\operatorname{div}\left(|D w|^{p-2} D w\right)=0 \\
w(x, \tau)=\left\{\begin{array}{lc}
u(x, \tau), & |x|<R, \\
0, & |x| \geq R .
\end{array}\right.
\end{gathered}
$$

By the results of the previous paragraph

$$
f_{B_{R}} u(x, \tau) \leq \gamma\left\{\left(\frac{R^{p}}{T}\right)^{1 /(p-2)}+\left(\frac{T}{R^{p}}\right)^{N / p}[w(0, T)]^{\kappa / p}\right\}
$$

where $\gamma=\gamma(N, p)$.

Theorem 3 will follow from (5.21) and the next comparison lemma.

Lemma 5.3. $w(x, t) \leq u(x, t),(x, t) \in \mathbf{R}^{N} \times(\tau, T)$.

Proof. Set

$$
M_{\tau}=\|u(\cdot, \tau)\|_{\infty, B_{R}}=\|w(\cdot, \tau)\|_{\infty, \mathbf{R}^{N}}
$$

and consider the comparison function

$$
\begin{aligned}
\mathscr{B}_{k, 2 R}(x, t ; 0, \tau)= & k(2 R)^{N} S(t)^{-N / \kappa}\left\{1-\left(\frac{|x|}{S(t)^{1 / \kappa}}\right)^{p /(p-1)}\right\}_{+}^{(p-1) /(p-2)} \\
S(t) & =\left\{\tilde{\gamma}_{0} k^{p-2}(2 R)^{N(p-2)}(t-\tau)+(2 R)^{\kappa}\right\}, \\
\tilde{\gamma}_{0}(N, p) & =\kappa(p /(p-2))^{p-1}, \quad \kappa=N(p-2)+p, \\
k & =M_{\tau}\left(1-2^{-p /(p-1)}\right)^{-(p-1) /(p-2)},
\end{aligned}
$$

which satisfies (2.1) for $t>\tau$ and $\forall t \geq \tau$ is supported in the ball $|x|<S(t)^{1 / \kappa}$.

For $t=\tau$ and $|x|<R$

$$
\mathscr{B}_{k, 2 R}(x, \tau ; 0, \tau) \geq M_{\tau}, \quad|x|<R .
$$

Therefore since $w(x, \tau) \leq M_{\tau},|x|<R$, by the comparison principle

$$
w(x, t) \leq \mathscr{B}_{k, 2 R}(x, t ; 0, \tau) \quad \forall(x, t) \in \mathbf{R}^{N} \times(\tau, T) .
$$

Such a principle can be applied since $(x, t) \rightarrow w(x, t)$ and $(x, t) \rightarrow$ $\mathscr{B}_{k, 2 R}(x, t ; 0, \tau)$ are both solutions of $(2.1)$ with initial data in $L^{1}\left(\mathbf{R}^{N}\right)$. We 
conclude that the support of $x \rightarrow w(x, t)$ is contained in $|x|<2 S(T)^{1 / \kappa}$ $\forall t \in[\tau, T]$.

But then in the bounded domain

$$
Q_{r_{0}}^{T} \equiv B_{r_{0}} \times(\tau, T), \quad r_{0}=2 S(T)^{1 / \kappa},
$$

both $u$ and $w$ satisfy (2.1) and $w \leq u$ on the parabolic boundary of $Q_{r_{0}}^{T}$. Moreover $u, w$ are both $C^{1, \alpha}$ in $B_{r_{0}} \times[\tau+\varepsilon, T], \forall \varepsilon>0$ and $D u, D w \in$ $L^{p}\left(Q_{r_{0}}^{T}\right)$. The comparison principle can now be applied to conclude $w(x, t) \leq$ $u(x, t), \forall(x, t) \in \mathbf{R}^{N} \times(\tau, T]$.

We conclude this section by observing that Lemma 5.3 and (5.7) give the proof of Lemma 2.2.

5-(iii). The initial trace. Let $u \geq 0$ be a solution of (2.1) in $S_{T}$. By Theorem 3, $\forall R>0, \forall 0<\tau<T / 2$

$$
f_{B_{R}} u(x, \tau) d x<\gamma\left\{\left(\frac{R^{p}}{T}\right)^{1 /(p-2)}+\left(\frac{T}{R^{p}}\right)^{N / \kappa}[u(0, T)]^{\kappa / p}\right\}
$$

where $\gamma=\gamma(N, p)$. It follows that $\forall \varphi \in C_{0}\left(B_{R}\right)$

$$
\left|\int_{\mathbf{R}^{N}} \varphi(x) u(x, \tau) d x\right| \leq \gamma\|\varphi\|_{\infty, \mathbf{R}^{N}}
$$

where $\gamma=\gamma(N, p, R, T, u(0, T))$. Therefore $\{u(\cdot, \tau)\}$ is a net of equibounded linear operators in $\left(C_{0}\left(\mathbf{R}^{N}\right)\right)^{*}$ and for a subnet indexed with $\tau^{\prime}$

$$
\lim _{\tau^{\prime} \backslash 0} \int_{\mathbf{R}^{N}} u\left(x, \tau^{\prime}\right) \varphi(x) d x=\int_{\mathbf{R}^{N}} \varphi d \mu
$$

where $\mu$ is a $\sigma$-finite Borel measure in $\mathbf{R}^{N}$. We prove uniqueness of $\mu$. Suppose that for two subnets $\left\{\tau^{\prime}\right\},\left\{\tau^{\prime \prime}\right\} \searrow 0$

$$
u\left(\cdot, \tau^{\prime}\right) \rightarrow \mu, \quad u\left(\cdot, \tau^{\prime \prime}\right) \rightarrow \nu, \quad \mathscr{D}^{\prime}\left(\mathbf{R}^{N}\right)
$$

and $\mu \neq \nu$.

From (5.22) $\forall \tau \in(0, T / 2)$

$$
\left(f_{B_{R}} u(x, \tau) d x\right)^{p-2} R^{-p} \leq C^{*}
$$

where $C^{*}=C^{*}(R, T, u(0, T))$ and from Lemma 2.2, $\forall \varepsilon>0, \forall R>0, \forall \tau \in$ $(0, T / 2)$

$$
\begin{aligned}
f_{B_{(1+\varepsilon) R}} u(x, t) d x \geq f_{B_{R}} u(x, \tau) d x\left((1+\varepsilon)^{-N}-\frac{\gamma}{\varepsilon}\left(C^{*} t\right)^{1 / \kappa}\right) \\
\forall 0<t-\tau<\gamma_{0} / C^{*}, t<T .
\end{aligned}
$$

Letting $\tau \searrow 0$ along $\left\{\tau^{\prime}\right\}$ we get

$$
f_{B_{(1+\varepsilon) R}} u(x, t) d x \geq f_{B_{R}} d \mu\left((1+\varepsilon)^{-N}-\frac{\gamma}{\varepsilon}\left(C^{*} t\right)^{1 / \kappa}\right) .
$$


We now let $t \searrow 0$ along $\left\{\tau^{\prime \prime}\right\}$. This gives

$$
f_{B_{(1+\varepsilon) R}} d \nu \geq(1+\varepsilon)^{-N} f_{B_{R}} d \mu, \quad \forall \varepsilon \in(0,1) .
$$

Letting $\varepsilon \rightarrow 0$ and interchanging the roles of $\mu$ and $\nu$, we find

$$
\int_{B_{\rho}} d \mu=\int_{B_{\rho}} d \nu, \quad \forall \rho>0 .
$$

By translation this must hold for every ball centered at any point of $\mathbf{R}^{N}$, and therefore $\mu \equiv \nu$.

\section{UNIQUENESS}

Let $u, v$ be two solutions of $(2.1)$ in $S_{T}, 0<T<\infty$, satisfying

$$
\sup _{0<t<T}\|\| u(\cdot, t) \mid\left\|_{r}+\sup _{0<t<T}\right\| v(\cdot, t)\|\|_{r} \equiv \Lambda<\infty,
$$

for some $r>0$, and

$$
|u-v|(\cdot, t) \rightarrow 0 \quad \text { in } L_{\text {loc }}^{1}\left(\mathbf{R}^{N}\right) \text { as } t \searrow 0 .
$$

Setting $w=u-v, w$ satisfies

$$
w_{t}-\left(a^{i, j}(x, t) w_{x_{i}}\right)_{x_{j}}=0 \text { in } S_{T}
$$

where

$$
\begin{aligned}
& a^{i, j}(x, t)=\left(\int_{0}^{1}|D(s u+(1-s) v)|^{p-2} d s\right) \delta_{i j} \\
& \quad+(p-2) \int_{0}^{1}|D(s u+(1-s) v)|^{p-4}(s u+(1-s) v)_{x_{i}}(s u+(1-s) v)_{x_{j}} d s .
\end{aligned}
$$

The matrix $\left(a^{i, j}\right)$ is positive semidefinite and $\forall \xi \in \mathbf{R}^{N}, \forall(x, t) \in S_{T}$

$$
\left\{\begin{array}{l}
a_{0}(x, t)|\xi|^{2} \leq a^{i, j}(x, t) \xi_{i} \xi_{j} \leq(p-1) a_{0}(x, t)|\xi|^{2}, \\
a_{0}(x, t)=\int_{0}^{1}|D(s u+(1-s) v)|^{p-2} d s, \quad(x, t) \in S_{T} .
\end{array}\right.
$$

We claim that $u$ and $v$ satisfy all the estimates of Theorem 1 with \|\|$u\|\|_{r}$ replaced by $\Lambda$, within the strip $S_{T_{0}}$, where

$$
T_{0}=\min \left\{T ; C_{0} \Lambda^{-(p-2)}\right\}
$$

where $C_{0}$ is introduced in Theorem 1 .

In particular, (2.10) takes the form

$$
\|u(\cdot, t)\|_{\infty, B_{\rho}} \leq C_{2} t^{-N / \kappa} \rho^{p /(p-2)} \Lambda^{p / \kappa}, \quad \forall \rho \geq r, 0<t<C_{0} \Lambda^{-(p-2)} .
$$

Also the analog of $(2.11)$ is

(6.8) $\|D u(\cdot, t)\|_{\infty, B_{\rho}} \leq C_{3} t^{-(N+1) / \kappa} \rho^{2 / p-2} \Lambda^{2 / \kappa}, \quad \forall \rho \geq r, 0<t<C_{0} \Lambda^{-(p-2)}$.

Estimates (6.7) and (6.8) will be proved in the next section (see Remark 7.1). Here we will proceed assuming them and observing that it will suffice to prove uniqueness within $S_{T_{0}}$. 
6-(i). Preliminaries. For $\alpha>0$ set

$$
A_{\alpha}(x)=\left(1+|x|^{p}\right)^{-\alpha}
$$

and define for $t \in\left(0, T_{0}\right)$

$$
h_{\alpha}(t)=\sup _{0<\tau \leq t} \int_{\mathbf{R}^{N}} u(x, \tau) A_{\alpha}(x) d x .
$$

If $t \in\left(0, T_{0}\right)$ and

$$
\alpha>\kappa / p(p-2),
$$

by Lemma 1.2 of [5] and (6.10), $h_{\alpha}(t)$ is finite and

$$
h_{\alpha}(t)<C(\alpha) \Lambda, \quad t \in\left(0, T_{0}\right),
$$

where $C(\alpha)=C(\alpha, N, p)$.

From now on we shall take $\alpha$ satisfying (6.11).

Lemma 6.1. There exists a constant $\gamma=\gamma(\Lambda, N, p)$ such that

$$
\int_{0}^{t} \int_{\mathbf{R}^{N}}|D u|^{p-1} A_{\alpha+1 / p} d x d \tau \leq \gamma t^{1 / \kappa}, \quad t \in\left(0, T_{0}\right) .
$$

Proof. In the weak formulation (2.17) take the test function

$$
(t-\varepsilon)_{+}^{1 / p} u^{1-2 / p}\left(A_{\alpha+1 / p}^{1 / p} \zeta\right)^{p}
$$

where $x \rightarrow \zeta(x)$ is the usual cutoff function in $B_{\rho}$. After a Steklov averaging process and standard calculations, we obtain

$$
\begin{aligned}
& \int_{\varepsilon}^{t} \int_{B_{\rho}}(\tau-\varepsilon)^{1 / p} \frac{|D u|^{p}}{u^{2 / p}} A_{\alpha+1 / p} \zeta^{p} d x d \tau \\
& \quad \leq \gamma \int_{\varepsilon}^{t} \int_{B_{\rho}}(\tau-\varepsilon)^{1 / p} u^{(p-2) / p} u^{p-1}\left|D\left(A_{\alpha+1 / p}^{1 / p} \zeta\right)\right|^{p} d x d \tau \\
& \quad+\gamma \int_{\varepsilon}^{t} \int_{B_{\rho}}(\tau-\varepsilon)^{1 / p-1} u^{(p-2) / p} A_{1 / p} u A_{\alpha} d x d \tau=J_{\rho}^{(1)}+J_{\rho}^{(2)}
\end{aligned}
$$

As for $J_{\rho}^{(2)}$, we have

$$
J_{\rho}^{(2)} \leq \gamma \int_{\varepsilon}^{t}(\tau-\varepsilon)^{1 / \kappa-1} \int_{B_{\rho}} \tau^{N(p-2) / p} \frac{|u(x, \tau)|^{(p-2) / p}}{\left(1+|x|^{p}\right)^{1 / p}} u(x, \tau) A_{\alpha}(x) d x d \tau,
$$

so that by (6.6) and (6.12)

$$
J_{\rho}^{(2)} \leq \gamma(t-\varepsilon)_{+}^{1 / \kappa}, \quad \forall \rho \geq r .
$$

We estimate $J_{\rho}^{(1)}$.

$$
\begin{aligned}
J_{\rho}^{(1)} \leq & \gamma \int_{\varepsilon}^{t} \int_{B_{\rho}}(\tau-\varepsilon)^{1 / p} u^{(p-2) / p} u^{p-1} A_{\alpha+1 / p}|D \zeta|^{p} d x d \tau \\
& +\gamma \int_{\varepsilon}^{t} \int_{B_{\rho}}(\tau-\varepsilon)^{1 / p} u^{(p-2) / p} u^{p-1}\left|D A_{\alpha+1 / p}^{1 / p}\right|^{p} d x d \tau \\
& =J_{\rho}^{(1,1)}+J_{\rho}^{(1,2)} .
\end{aligned}
$$


Since

$$
\left|D A_{\alpha+1 / p}^{1 / p}\right| \leq \gamma\left|A_{\alpha / p+1 / p^{2}+1 / p}\right|^{p} \leq \gamma A_{\alpha} A_{1 / p} A_{1},
$$

by (6.6) and (6.12),

$$
\begin{aligned}
J_{\rho}^{(1,2)} & \leq \gamma \int_{\varepsilon}^{t} \int_{B_{\rho}}(\tau-\varepsilon)^{1 / p} u^{(p-2) / p} A_{1 / p}(x) u^{p-2} A_{1}(x) u A_{\alpha} d x d \tau \\
& \leq \gamma h_{\alpha}(t) \int_{\varepsilon}^{t}(\tau-\varepsilon)^{1 / p-N(p-2) / \kappa p-N(p-2) / \kappa} d \tau \\
& \leq \gamma(t-\varepsilon)^{1 / \kappa}, \quad \forall \rho \geq r .
\end{aligned}
$$

As for $J_{\rho}^{(1,1)}$, since $|D \zeta| \leq 2 / \rho$, again by (6.6) and (6.12)

$$
J_{\rho}^{(1,1)} \leq \gamma \int_{\varepsilon}^{t}(\tau-\varepsilon)^{1 / \kappa-1} d \tau h_{\alpha}(t) \leq \gamma(t-\varepsilon)^{1 / \kappa} .
$$

Combining these estimates in (6.13),

$$
\int_{\varepsilon}^{t} \int_{B_{\rho}}(\tau-\varepsilon)^{1 / p} \frac{|D u|^{p}}{u^{2 / p}} A_{\alpha+1 / p} d x d \tau \leq \gamma(t-\varepsilon)^{1 / \kappa}
$$

$\forall \rho \geq r, \forall t \in\left(\varepsilon, T_{0}\right)$, where we have changed $\rho$ into $2 \rho$.

Next, $\forall \varepsilon \in(0, t), t \in\left(0, T_{0}\right)$

$$
\begin{aligned}
\int_{\varepsilon}^{t} \int_{B_{\rho}}|D u|^{p-1} A_{\alpha+1 / p} d x d \tau \\
=\int_{\varepsilon}^{t} \int_{B_{\rho}}\left\{(\tau-\varepsilon)^{(p-1) / p^{2}} \frac{|D u|^{p-1}}{\left.u^{2(p-1) / p^{2}} A_{\alpha+1 / p}^{(p-1) / p}\right\}}\right. \\
\cdot\left\{(\tau-\varepsilon)^{-(p-1) / p^{2}} u^{\left(2 / p^{2}\right)(p-1)} A_{\alpha+1 / p}^{1 / p}\right\} d x d \tau \\
\leq\left(\int_{\varepsilon}^{t} \int_{B_{\rho}}(t-\varepsilon)^{1 / p} \frac{|D u|^{p}}{\left.u^{2 / p} A_{\alpha+1 / p} d x d \tau\right)^{(p-1) / p}}\right. \\
\cdot\left(\int_{\varepsilon}^{t} \int_{B_{\rho}}(\tau-\varepsilon)^{-(p-1) / p} u^{(p-2) / p} A_{1 / p} u A_{\alpha} d x d \tau\right)^{1 / p} \\
\leq \gamma(t-\varepsilon)^{(1 / \kappa)(p-1) / p}\left(\int_{\varepsilon}^{t}(\tau-\varepsilon)^{1 / \kappa-1} h_{\alpha}(\tau) d \tau\right)^{1 / p} \\
\leq \gamma(t-\varepsilon)^{1 / \kappa}, \quad \forall \rho>r, \forall t \in\left(0, T_{0}\right) .
\end{aligned}
$$

Lemma 6.2. There exists a constant $\gamma=\gamma(\Lambda, N, p)$ such that if $w(\cdot, t) \rightarrow 0$ in $L_{\text {loc }}^{1}\left(\mathbf{R}^{N}\right)$ as $t \searrow 0$,

$$
\int_{\mathbf{R}^{N}}|w(x, t)| A_{\alpha}(x) d x \leq \gamma t^{1 / \kappa}, \quad 0<t<T_{0} .
$$

Proof. In the weak formulation of (6.3) take the test function $x \rightarrow A_{\alpha}(x) \zeta(x)$, where $\zeta$ is the usual cutoff function in $B_{\rho}$. By working separately with $w^{+}$ 
and $w^{+}$(both weak subsolutions of (6.3)) and using the assumptions of the lemma we deduce

$$
\begin{aligned}
\int_{B_{\rho}}|w(x, t)| A_{\alpha}(x) \zeta(x) d x \leq & \int_{0}^{t} \int_{B_{\rho}}(|D u|+|D v|)^{p-1}\left|D A_{\alpha} \zeta\right| d x d \tau \\
\leq & \gamma \int_{0}^{t} \int_{B_{\rho}}(|D u|+|D v|)^{p-1} A_{\alpha}|D \zeta| d x d \tau \\
& +\gamma \int_{0}^{t} \int_{B_{\rho}}(|D u|+|D v|)^{p-1}\left|D A_{\alpha}\right| d x d \tau
\end{aligned}
$$

In the last integral $\left|D A_{\alpha}\right| \leq \gamma A_{\alpha+1 / p}$ and in the first integral, since $|D \zeta|=0$ on $|x|<\rho / 2$, we have $A_{\alpha}|D \zeta| \leq \gamma A_{\alpha+1 / p}$.

Therefore letting $\rho \rightarrow \infty$

$$
\int_{\mathbf{R}^{N}}|w(x, t)| A_{\alpha}(x) d x \leq \gamma \int_{0}^{t} \int_{\mathbf{R}^{N}}(|D u|+|D v|)^{p-1} A_{\alpha+1 / p} d x d \tau
$$

and the conclusion follows from Lemma 6.1.

Lemma 6.3. If $w(\cdot, t) \rightarrow 0$ in $L_{\mathrm{loc}}^{1}\left(\mathbf{R}^{N}\right)$ as $t \rightarrow 0$, then

$$
w(\cdot, t) \rightarrow 0 \text { in } L_{\text {loc }}^{1+\varepsilon}\left(\mathbf{R}^{N}\right) \quad \text { as } t \rightarrow 0, \forall \varepsilon \in(0,1 / N) .
$$

Proof. Let $\varepsilon \in(0,1 / N)$ be fixed. Then $\forall t \in\left(0, T_{0}\right)$

$$
\int_{\mathbf{R}^{N}}|w(x, t)|^{1+\varepsilon} A_{\alpha+\varepsilon /(p-2)}(x) d x \leq \int_{\mathbf{R}^{N}}|w(x, t)|^{\varepsilon} A_{\varepsilon /(p-2)}(x)|w(x, t)| A_{\alpha}(x) d x .
$$

By (6.6) $|w(x, t)|^{\varepsilon} A_{\varepsilon /(p-2)}(x) \leq \gamma t^{-N \varepsilon / \kappa}$, so that

$$
\begin{aligned}
\int_{\mathbf{R}^{N}}|w(x, t)|^{1+\varepsilon} A_{\alpha+\varepsilon /(p-2)}(x) d x & \leq \gamma t^{-N \varepsilon / \kappa} \int_{\mathbf{R}^{N}}|w(x, t)| A_{\alpha}(x) d x \\
& \leq \gamma t^{(1 / \kappa)(1-N \varepsilon)} \text { by Lemma 6.2. }
\end{aligned}
$$

For $\rho \geq 1$

$$
\begin{aligned}
\int_{B_{\rho}}|w(x, t)|^{1+\varepsilon} d x & \leq \gamma \rho^{(\alpha+\varepsilon /(p-2)) p} \int_{\mathbf{R}^{N}}|w(x, t)|^{1+\varepsilon} A_{\alpha+\varepsilon /(p-2)}(x) d x \\
& \leq \gamma(\Lambda, N, p, \rho) t^{(1 / \kappa)(1-N \varepsilon)} .
\end{aligned}
$$

6-(ii). Proof of Theorem 4. We are now in the position to prove our uniqueness theorem. In (6.3) we may assume without loss of generality that $w \geq 0$. In its weak formulation we take test functions

$$
(w+\eta)^{\varepsilon}\left(A_{\alpha}^{1 / 2} \zeta\right)^{2}, \quad \varepsilon \in(0,1 / N), \quad \eta \in(0,1)
$$

Since $w(\cdot, t) \rightarrow 0$ in $L_{\text {loc }}^{1}\left(\mathbf{R}^{N}\right)$ a standard Steklov averaging process gives that this is an admissible test function. Integrating over $B_{\rho} \times(\eta, t), 0<\eta<t \leq T_{0}$, 
we obtain

(6.15)

$$
\begin{aligned}
\frac{1}{1+\varepsilon} & \int_{B_{\rho}(t)}(w+\eta)^{1+\varepsilon} A_{\alpha} \zeta^{2} d x+\varepsilon \int_{\eta}^{t} \int_{B_{\rho}} a_{0}(x, \tau) \frac{|D w|^{2}}{(w+\eta)^{1-\varepsilon}}\left(A_{\alpha}^{1 / 2} \zeta\right)^{2} d x d \tau \\
\leq & \frac{1}{1+\varepsilon} \int_{B_{\rho}(\eta)}(w+\eta)^{1+\varepsilon} A_{\alpha} \zeta^{2} d x \\
& +\gamma \int_{\eta}^{t} \int_{B_{\rho}} a_{0}(x, \tau) \frac{|D w|}{(w+\eta)^{(1-\varepsilon) / 2}}(w+\eta)^{(1+\varepsilon) / 2}\left(A_{\alpha}^{(1 / 2)} \zeta\right)\left|D\left(A_{\alpha}^{1 / 2} \zeta\right)\right| d x d \tau,
\end{aligned}
$$

where $a_{0}(x, t)$ has been defined in (6.5).

By the Schwarz inequality the last integral is majorized by

$$
\begin{aligned}
& \frac{\varepsilon}{2} \int_{\eta}^{t} \int_{B_{\rho}} a_{0}(x, \tau) \frac{|D w|^{2}}{(w+\eta)^{1-\varepsilon}}\left(A_{\alpha}^{1 / 2} \zeta\right)^{2} d x d \tau \\
& \quad+\gamma(\varepsilon) \int_{\eta}^{t} \int_{B_{\rho}} a_{0}(x, \tau)(w+\eta)^{1+\varepsilon}\left(A_{\alpha}|D \zeta|^{2}+\left|D A_{\alpha}^{1 / 2}\right|^{2}\right) d x d \tau
\end{aligned}
$$

We absorb the integral involving $|D w|^{2}$ on the left-hand side of (6.15) and drop the resulting nonnegative term. Finally, we observe that by the definition of $A_{\alpha}$ and the structure of $x \rightarrow \zeta(x)$ we have

$$
A_{\alpha}|D \zeta|^{2}+\left|D A_{\alpha}^{1 / 2}\right|^{2} \leq \gamma A_{\alpha}(x) A_{2 / p}(x)
$$

Carrying these remarks in (6.15) we obtain

$$
\begin{aligned}
& \int_{B_{\rho}(t)}(w+\eta)^{1+\varepsilon} A_{\alpha} \zeta^{2} d x \leq \int_{B_{\rho}(\eta)}(w+\eta)^{1+\varepsilon} A_{\alpha}(x) d x \\
& +\gamma \int_{\eta}^{t} \int_{B_{\rho}} a_{0}(x, \tau) A_{2 / p}(x)(w+\eta)^{1+\varepsilon} A_{\alpha}(x) d x d \tau .
\end{aligned}
$$

Next by (6.5) and (6.8)

$$
a_{0}(x, \tau) A_{2 / p}(x) \leq \gamma \frac{|x|^{2}}{\left(1+|x|^{p}\right)^{2 / p}} \Lambda^{(2 / \kappa)(p-2)} \tau^{-((N+1) / \kappa)(p-2)} .
$$

Substitute this last estimate in (6.16) and let $\eta \rightarrow 0$ for $\rho \geq 1$ fixed so that by Lemma 6.3

$$
\int_{B_{\rho}(\eta)}(w+\eta)^{1+\varepsilon} A_{\alpha}(x) d x \rightarrow 0 \text { as } \eta \rightarrow 0 .
$$

Then we let $\rho \rightarrow \infty$. The net result is

$$
\int_{\mathbf{R}^{N}}|w(x, t)|^{1+\varepsilon} A_{\alpha}(x) d x \leq \gamma \int_{0}^{t} \tau^{-((N+1) / \kappa)(p-2)} \int_{\mathbf{R}^{N}}|w(x, \tau)|^{1+\varepsilon} A_{\alpha}(x) d x .
$$

Since $\tau^{-((N+1) / \kappa)(p-2)}$ is integrable, this implies

$$
t \rightarrow \int_{\mathbf{R}^{N}}|w(x, t)|^{1+\varepsilon} A_{\alpha}(x) d x \equiv 0,
$$


by Gronwall's lemma, provided

$$
t \rightarrow \int_{\mathbf{R}^{N}}|w(x, t)|^{1+\varepsilon} A_{\alpha}(x) d x \in L^{\infty}\left(0, T_{0}\right) .
$$

Now the parameter $\alpha$ in the calculations above is arbitrary and restricted only by (6.11). If $\alpha$ is replaced by $\alpha+\varepsilon /(p-2)$, then Lemma 6.3 and its proof ensure the $L^{\infty}\left(0, T_{0}\right)$ requirement and the theorem follows.

\section{Proof of TheOrem 5}

We let $u$ be a nonnegative solution of (2.1) in $S_{T}$ for some $T>0$, satisfying

$$
\sup _{0<t<T}\|u(\cdot, t) \mid\|_{r} \equiv \Lambda<\infty \quad \text { for some } r>0 .
$$

The solution here is meant in the sense of (2.15), (2.16) of $\S 2$-(ii). The following proposition holds for any solution of (2.1) in $S_{T}$ with no sign restriction.

Proposition 7.1. Let $u$ be a weak solution of (2.1) in $S_{T}$ in the sense of (2.15), (2.16), and let (7.1) hold. There exist constants $C_{i}=C_{i}(N, p), i=0,2,3,7$, such that setting

$$
T_{0}=\min \left\{T ; C_{0} \Lambda^{-(p-2)}\right\}
$$

the following estimates hold $\forall \rho \geq 0, \forall 0<t \leq T$ :

$$
\begin{gathered}
\|u(\cdot, t)\|_{\infty, B_{\rho}} \leq C_{2} t^{-N / \kappa} \rho^{p /(p-2)} \Lambda^{p / \kappa}, \\
\|D u(\cdot, t)\|_{\infty, B_{\rho}} \leq C_{3} t^{-(N+1) / \kappa} \rho^{p /(p-2)} \Lambda^{2 / \kappa}, \\
\int_{0}^{t} \int_{B_{\rho}}|D u|^{p-1} d x d \tau \leq C_{7} t^{1 / \kappa} \rho^{1+(p-2) / \kappa} \Lambda^{1+(p-2) / \kappa} .
\end{gathered}
$$

Here the constants $C_{i}, i=0,2,3,7$, are the same as those in Theorem 1. Proof. Fix $\varepsilon \in\left(0, T_{0} / 4\right)$ and view $(x, t) \rightarrow u(x, t)$ as solution of

$$
\begin{gathered}
u_{t}-\operatorname{div}|D u|^{p-2} D u=0 \text { in } \mathbf{R}^{N} \times\left(\varepsilon, T_{0}\right], \\
x \rightarrow u(x, \varepsilon) \text { as initial datum. }
\end{gathered}
$$

The estimates of $\S 3$, regarding (2.10), have been derived under the assumption that

$$
u_{0} \in C_{0}^{\infty}\left(\mathbf{R}^{N}\right) \text {. }
$$

This was needed to ensure existence of a solution $u$ satisfying

$$
x \rightarrow u(x, t) \in L^{\infty}\left(\mathbf{R}^{N}\right), \quad \forall t \geq 0 .
$$

This in turn was used to ensure that $t \rightarrow \phi(t)$ in (3.10) is well defined. Therefore (7.3) will follow from exactly the same proof of (2.10), whence we show that

$$
\sup _{\rho \geq r} \frac{\|u(\cdot, t)\|_{\infty, B_{\rho}}}{\rho^{p /(p-2)}}<\infty \quad \forall t \in\left(0, T_{0}\right), \text { for some } r>0 .
$$


Analogously, the gradient estimates (7.4), (7.5) can be derived exactly as in the proofs of $\S \S 3,4$, whence we show that

$$
\sup _{\rho \geq r} \frac{\|D u(\cdot, t)\|_{\infty, B_{\rho}}}{\rho^{2 /(p-2)}}<\infty, \quad \forall t \in\left(0, T_{0}\right), \text { for some } r>0 .
$$

The qualitative information in (7.9), (7.10) can be derived from the quantitative datum (7.1) as follows. Let

$$
Q_{d} \equiv B_{d} \times\left\{-d^{p}, 0\right\}, \quad d \in(0,4),
$$

and let $U$ be a local weak solution of (2.1) in $Q_{4}$. By the local estimates of Porzio-Vincenzotti (see [17]), $\forall \nu>0$, there exists a constant $\gamma=\gamma(N, p, \nu)$ such that

$$
\|U\|_{\infty, Q_{1}} \leq \gamma\left(\iint_{Q_{2}}|U|^{p-2+\nu} d x d \tau\right)^{1 / \nu} .
$$

Take $\nu=(2 N+p) / N$ in (7.11) and observe that by the embedding of $\S 2$ and the equation, we have

$$
\iint_{Q_{r}}|U|^{p(N+1) / N} d x d \tau \leq \frac{\gamma}{\sigma^{\theta}}\left(\iint_{Q_{(1+\sigma) r}}|U|^{p} d x d \tau\right)\left(\sup _{-4^{p} \leq t \leq 0} \int_{B_{4}(t)}|U| d x\right)^{p / N}
$$

$\forall r>0, \forall \sigma \in(0,1)$, where $\gamma, \theta=\gamma, \theta(N, p)$. From this, Hölder and Young's inequalities, $\forall \delta \in(0,1)$

$$
\begin{aligned}
\iint_{Q_{r}}|U|^{p(N+1) / N} d x d \tau \leq & \delta \iint_{Q_{(1+\sigma) r}}|U|^{p(N+1) / N} d x d \tau \\
& +\frac{\gamma(N, p, \delta)}{\sigma^{\theta(N+1)}}\left(\sup _{-4 p \leq t \leq 0} \int_{B_{4}(t)}|U| d x\right)^{p(N+1) / N} .
\end{aligned}
$$

By a standard interpolation process

$$
\iint_{Q_{2}}|U|^{p(N+1) / N} d x d \tau \leq \gamma(N, p)\left(\sup _{-4^{p} \leq t \leq 0} \int_{B_{4}(t)}|U| d x\right)^{p(N+1) / N}
$$

which combined with (7.10) with $\nu=(2 N+p) / N$ yields

$$
\|U\|_{\infty, Q_{1}} \leq \gamma\left(\sup _{-4^{p} \leq t \leq 0} \int_{B_{4}(t)}|U| d x\right)^{p(N+1) /(2 N+p)}, \quad \gamma=\gamma(N, p) .
$$

Now let $u$ be a weak solution of (7.6) in $B_{4 \varepsilon} \times\left(\varepsilon, T_{0}\right)$. Then the rescaled function

$$
U(x, t) \equiv\left(\frac{-\varepsilon+T_{0}}{(4 \rho)^{p}}\right)^{1 /(p-2)} u\left(x \rho, t\left(\frac{\varepsilon-T_{0}}{4^{p}}\right)+\varepsilon\right)
$$

is a solution of (7.6) in $Q_{4}$. Therefore (7.12) and (7.1) imply

$$
\forall \frac{T_{0}}{4} \leq t \leq T_{0}, \quad \frac{\|u(\cdot, t)\|_{\infty, B_{\rho}}}{\rho^{p /(p-2)}} \leq \gamma T_{0}^{N /(2 N+p)} \Lambda^{p(N+1) /(2 N+p)} .
$$


Since the argument can be repeated with $T_{0}$ replaced by any $0<t<T_{0}$, (7.9) follows.

Turning to the proof of (7.10), if $U$ is as before, by Proposition 3.1 of [18] and the equation

$$
\begin{aligned}
\|D U\|_{\infty, Q_{1}} & \leq \gamma\left[\left(\iint_{Q_{2}}|D U|^{p} d x d \tau\right)^{1 / 2}+1\right] \\
& \leq \gamma\left[\left(\iint_{Q_{4}}|U|^{p} d x d \tau\right)^{1 / 2}+1\right] .
\end{aligned}
$$

Returning to $(x, t) \rightarrow u(x, t)$ via the indicated rescaling proves (7.10). We remark that (7.9), (7.10) are qualitative information needed in the proof of $\S \S 3$, 4. The arguments in these sections turn these qualitative information into the precise quantitative estimates of Proposition 7.1.

The proposition and the Harnack inequality prove Theorem 5.

Remark 7.1. Since Proposition 7.1 holds for solutions of (2.1) with no sign restriction, estimates $(6.7),(6.8)$ are valid and the proof of the uniqueness theorem is complete.

\section{REFERENCES}

1. N. D. Alikakos and R. Rostamian, Gradient estimates for degenerate diffusion equations II, Proc. Roy. Soc. Edinburgh Sect. A 91 (1981/82), 335-346.

2. D. G. Aronson, Widder's inversion theorem and the initial distribution problem, SIAM J. Math. Anal. 12, 4 (1981), 639-651.

3. D. G. Aronson and L. A. Caffarelli, The initial trace of a solution of the porous medium equation, Trans. Amer. Math. Soc. 3801 (1983), 351-366.

4. Ph. Bènilan and M. G. Crandall, Regularizing effects of homogeneous evolution equations, MRC Tech. Rep. 2076, Madison, Wis., 1980.

5. Ph. Bènilan, M. G. Crandall, and M. Pierre, Solutions of the porous medium equation in $\mathbf{R}^{N}$ under optimal conditions on initial values, Indiana Univ. Math. J. 33 (1984), 51-87.

6. B. E. J. Dahlberg and C. E. Kenig, Nonnegative solutions of the porous medium equation, Comm. Partial Differential Equations 9 (1984), 409-437.

7. E. DiBenedetto, On the local behaviour of solutions of degenerate parabolic equations with measurable coefficients, Ann. Scuola Norm. Sup. Pisa Cl. Sci. (4) 13, 3 (1986), 487-535.

8. _ Intrinsic Harnack type inequalities for solutions of certain degenerate parabolic equations, Arch. Rational Mech. Anal. 100 (1988), 129-147.

9. E. DiBenedetto and A. Friedman, Hölder estimates for nonlinear degenerate parabolic systems, J. Reine Angew. Math. 357 (1985), 1-22.

10. M. A. Herrero and J. L. Vazquez, Asymptotic behaviour of the solutions of a strongly nonlinear parabolic problem, Ann. Fac. Sci. Toulouse Math. 3 (1981), 113-127.

11. A. S. Kalashnikov, Cauchy's problem in classes of increasing functions for certain quasi-linear degenerate parabolic equations of the second order, Differencial' nye Uravnenija 9 (1973), 682-691.

12. __ On uniqueness conditions for the generalized solutions of the Cauchy problem for a class of quasi-linear degenerate parabolic equations, Differencial ' nye Uravnenija 9 (1973), 22072212. 
13. O. A. Ladyzenskaja, V. A. Solonnikov, and N. N. Ural' tseva, Linear and quasi-linear equations of parabolic type, Transl. Math. Monographs, vol. 23, Amer. Math. Soc., Providence, R. I., 1968.

14. A. N. Tychonov, Théorèmes d'unicité pour léquation de la chaleur, Mat. Sb. 42 (1935), 199216.

15. D. V. Widder, Positive temperatures in an infinite rod, Trans. Amer. Math. Soc. 55 (1944), 85-95.

16. G. I. Barenblatt, On some unsteady motions of a liquid or a gas in a porous medium, Prikl. Mat. Meh. 16 (1952), pp. 67-78.

17. M. Porzio and P. Vincenzotti, A priori bounds for weak solutions of certain degenerate parabolic equations (to appear).

18. Chen Ya-zhe and E. DiBenedetto, Boundary estimates for solutions of nonlinear degenerate parabolic systems, J. Reine Angew. Math. 395 (1989), 102-131.

Department of Mathematics, Northwestern University, Evanston, Illinois 60208

Departamento de Matemática Aplicada, Facultad de Matemáticas, Universidad ComPLUTENSE, 28040 MADRID, SPAIN 